\title{
Safe maneuvering envelope estimation based on a physical approach
}

\author{
Thomas J.J. Lombaerts* \\ German Aerospace Center DLR, Münchner Straße 20, 82234 Weßling, Germany \\ NASA Ames Research Center, Moffett Field, CA 94035, USA
}

Stefan R. Schuet ${ }^{\dagger}$ and Kevin R. Wheeler ${ }^{\ddagger}$ and Diana M. Acosta ${ }^{\ddagger}$ and John T. Kaneshige ${ }^{\ddagger}$

NASA Ames Research Center, Moffett Field, CA 94035, USA

\begin{abstract}
This paper discusses a computationally efficient algorithm for estimating the safe maneuvering envelope of damaged aircraft. The algorithm performs a robust reachability analysis through an optimal control formulation while making use of time scale separation and taking into account uncertainties in the aerodynamic derivatives. This approach differs from others since it is physically inspired. This more transparent approach allows interpreting data in each step, and it is assumed that these physical models based upon flight dynamics theory will therefore facilitate certification for future real life applications.
\end{abstract}

\section{Nomenclature}

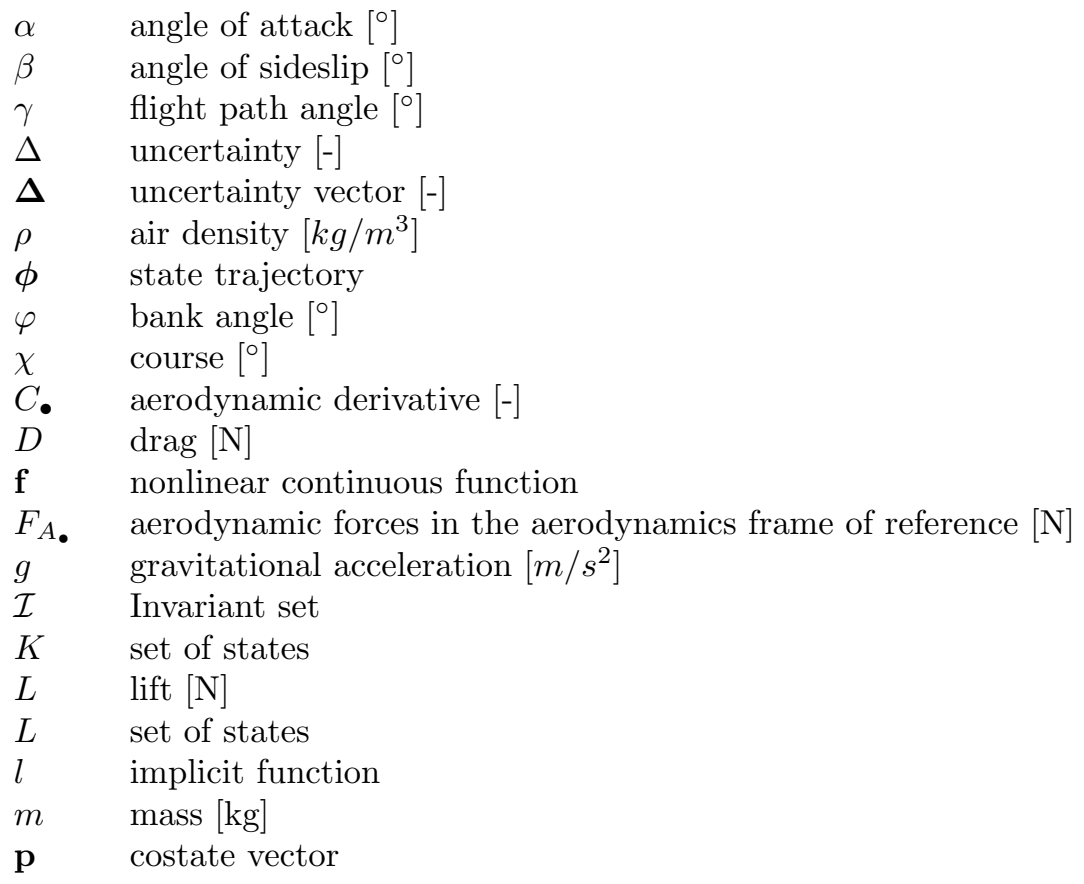

\footnotetext{
${ }^{*}$ Researcher at DLR, Institute of System Dynamics and Control, Department of Aircraft Dynamics, e-mail: thomas.lombaerts@dlr.de, currently Marie Curie Fellow and visiting researcher at NASA Ames Research Center, Intelligent Systems Division, Adaptive Control and Evolvable Systems (ACES) Group, e-mail: thomas.lombaerts@nasa.gov, AIAA Member.

${ }^{\dagger}$ Researcher at NASA Ames Research Center, Intelligent Systems Division, Physics based methods Group

$\ddagger$ Researcher at NASA Ames Research Center, Intelligent Systems Division, Adaptive Control and Evolvable Systems (ACES) Group, AIAA member.
} 


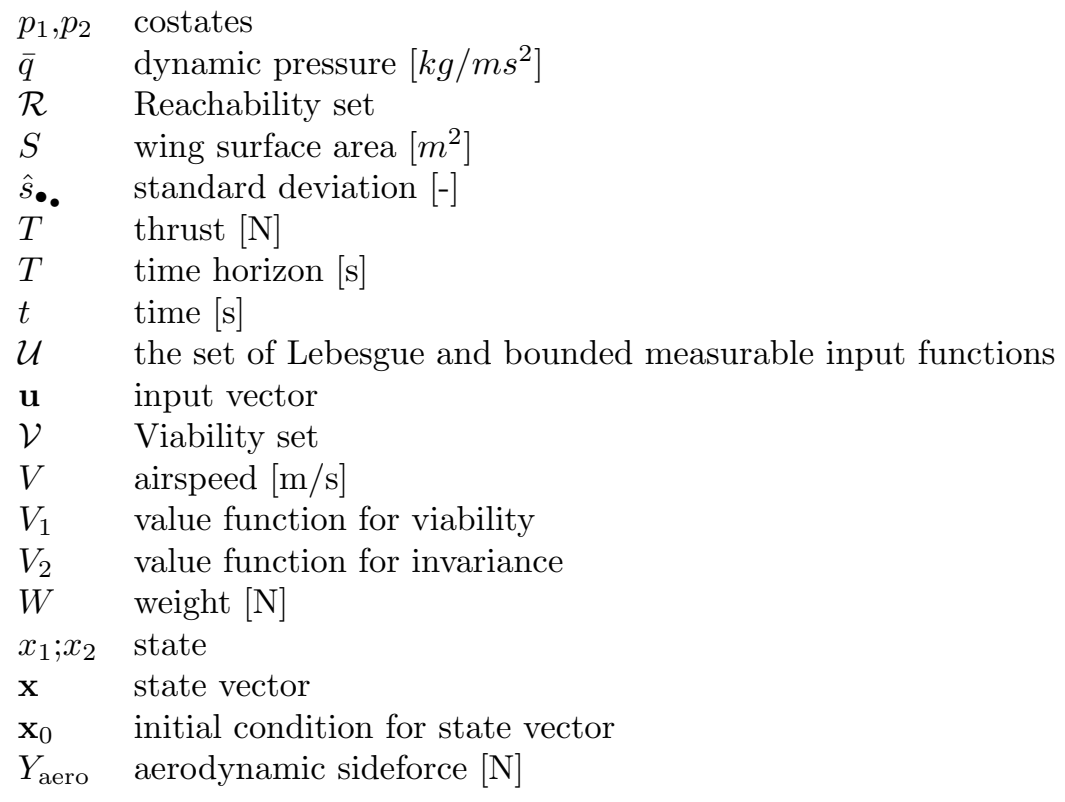

\section{Introduction}

CAFETY is of paramount importance in all transportation systems, but especially in civil aviation. ThereOfore, in civil aviation, many developments focus on improving safety levels and reducing the risks that critical failures occur. In a recent study by CAST/ICAO, it can be observed that loss of control in flight (LOC-I) is the most frequent primary accident cause. This study is based on a statistical analysis of aircraft accidents between 2002 and 2011, and indicates that this category accounts for as much as $23 \%$ of all fatal aircraft accidents and involves most fatalities. 1 Benefit can be gained by developing technology which prevents these LOC-I accidents. From a flight dynamics point of view, with the technology and computing power available on this moment, it might have been possible to recover some of the aircraft in the accident category described above on the condition that non-conventional control strategies would have been applied. These non-conventional control strategies involve the so-called concept of fault tolerant flight control (FTFC), where the control system is capable to detect and adapt for changes in the aircraft behavior.

One FTFC strategy option is using a model based control routine. Previous research focused on a physical model approach.2 In this setup, experiments have shown that not only a reconfiguring controller is needed, but also some form of flight envelope protection, which prevents the airplane from leaving the safe flight envelope and loosing control in flight!2 The main challenge in this context is determining the new bounds of the safe flight envelope after failure, which are then used by the envelope protection algorithm. $\underline{3}$

Alternative and complementary research approaches for the purpose of loss of control prevention and prediction are among others passive adaptive control, $\stackrel{4}{4}$ data-based predictive control $\stackrel{5}{ }$ and real-time optimal envelope limit estimation. 6

Determination of the flight envelope has been done in the literature through various methods. The most straightforward methods include wind tunnel testing, flight test experiments and high-fidelity model-based computation of attainable equilibrium sets or achievable trim points, $\frac{7}{8[10}$ possibly with bifurcation analysis $11[2]$ or a vortex lattice algorithm combined with an extended Kalman filter 13 More complex methods include formulating flight envelope estimation as a reachability problem and solving this with level set methods and Hamilton-Jacobi equations, 14, 15, 16, 17, 18 possibly with time scale separation 19 or semi-Lagrangian level sets. 20 Alternative methods rely on linearization and region of attraction analysis, ${ }^{21}$ determining controllability/maneuverability limits in a quaternion-based control architecture ${ }^{22}$ or robustness analysis for determination of reliable flight regimes.23 An approach suggested by Boeing uses Control-Centric Modeling, dynamic flexible structure and load models. 24 In the frequency domain, stability margins can be estimated in real time via nonparametric system identification.25 A complete different alternative is relying on the bio-immune system metaphor in combination with artificial intelligence.26 27.28 More focused techniques inspired by flight dynamics exist as well, such as determining the minimum lateral control speed ${ }^{29}$ Online methods are preferable from an operational point of view. For fault tolerant systems, the adaptive flight 
envelope estimation must take into account damage information which will possibly restrict the safe envelope significantly.

From the perspective of the physical approach, the preferred interpretation of the safe maneuvering envelope considers reachability from the trim envelope. The stable and controllable trim envelope is considered an a-priori safe set. The backwards reachable set is defined as the set of states from where (at least one point in) the trim envelope can be reached. The forwards reachable set is defined as the set of states which can be reached from (at least one point in) the trim envelope. Then the safe maneuvering flight envelope is the cross section between the forwards and backwards reachable sets. This interpretation is illustrated in Fig. 1. In addition to the safe envelope, the backwards reachable set is considered as the survivable flight envelope. After an upset due to damage, turbulence, a wake encounter etc., it is possible to bring the aircraft back to a safe trim condition as long as the current flight condition is situated inside the backwards reachable set.

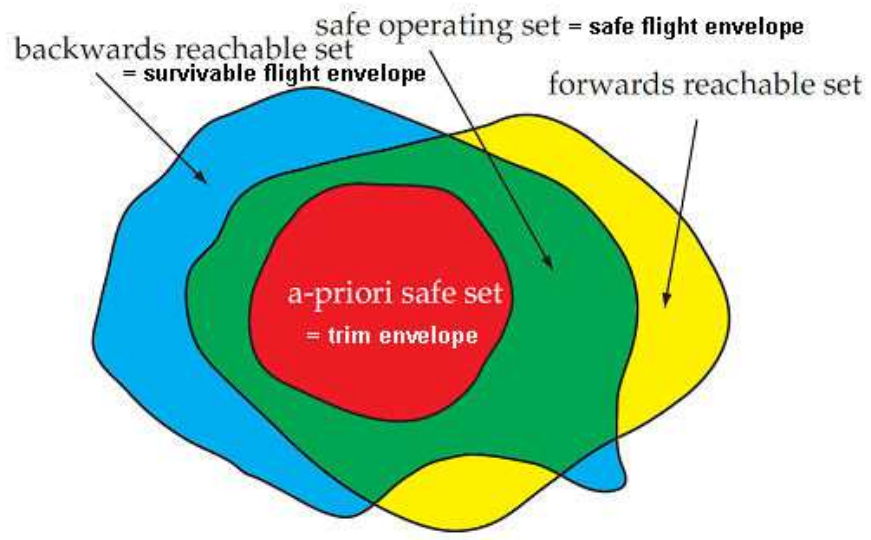

Figure 1. Safe maneuvering envelope as intersection between forwards and backwards reachability, source: van Oort 20

The aim is to perform a combined forward and backward reachability analysis from the trim envelope as efficiently as possible, for on-line implementations. Based upon previous research ${ }^{6}$ level set methods are an excellent candidate. Two of the major challenges are the computational load and how to cope with nonlinear systems with higher dimensions. In general, an increase in technology readiness level (TRL) is envisaged.

Nonlinear systems with higher dimensions can be simplified by considering the principle of time scale separation. ${ }^{19}$ The structure of time scale separation is analogous as applied for the fault tolerant control algorithm developed earlier. 2] The overview can be found in Fig. 2, which illustrates that a nine dimensional nonlinear problem is decoupled in three consecutive three dimensional optimization problems.

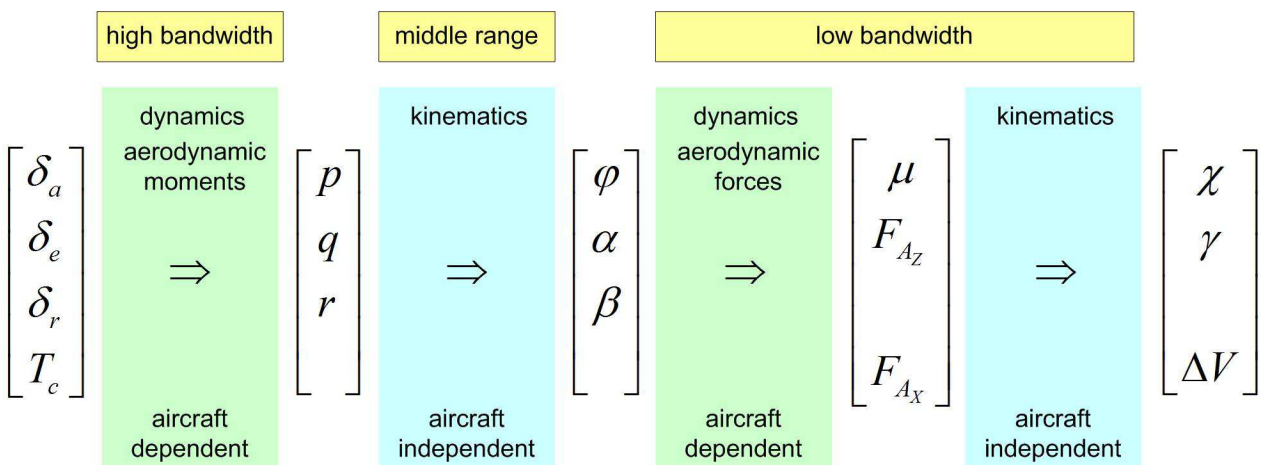

Figure 2. Separation of dynamics over high bandwidth, middle range and low bandwidth 


\section{Optimal Control Formulation}

It has been shown in the literature that maneuvering envelope estimation through reachability can be reformulated in the optimal control framework ${ }^{[14}$ Consider a continuous time control system:

$$
\dot{\mathbf{x}}=\mathbf{f}(\mathbf{x}, \mathbf{u})
$$

with $\mathbf{x} \in \mathbb{R}^{n}, \mathbf{u} \in U \subseteq \mathbb{R}^{m}, \mathbf{f}(\cdot, \cdot): \mathbb{R}^{n} \times U \rightarrow \mathbb{R}^{n}$, a function:

$$
l(\cdot): \mathbb{R}^{n} \rightarrow \mathbb{R}
$$

and an arbitrary time horizon $T \geq 0$. Let $\mathcal{U}_{\left[t, t^{\prime}\right]}$ denote the set of Lebesgue and bounded measurable functions from the interval $\left[t, t^{\prime}\right]$ to $U$. Define $\phi(\tau, t, \mathbf{x}, \mathbf{u}(\cdot))$ as the state trajectory. Given a set of states $K \subseteq \mathbb{R}^{n}$, a number of questions can be naturally formulated regarding the relation between the set $K$ and the state trajectories $\phi$ of Eq. (11) over the horizon T. Problems of interest include the following:

Viability: Does there exist a $\mathbf{u} \in \mathcal{U}_{[0, T]}$ for which the trajectory $\phi$ of the state $\mathbf{x}$ satisfies $\mathbf{x} \in K$ for all $t \in[0, T]$ ?

Invariance: Do the trajectories $\phi$ of the state $\mathbf{x}$ for all $\mathbf{u} \in \mathcal{U}_{[0, T]}$ satisfy $\mathbf{x} \in K$ for all $t \in[0, T]$ ?

Reachability: Does there exist a $\mathbf{u} \in \mathcal{U}_{[0, T]}$ and a $t \in[0, T]$ such that the trajectory $\phi$ of the state $\mathbf{x}$ satisfies $\mathrm{x} \in K$ ?

More generally, the following sets can be characterized:

$$
\begin{aligned}
\mathcal{V}(t, K) & =\left\{\mathbf{x} \in \mathbb{R}^{n} \mid \exists \mathbf{u} \in \mathcal{U}_{[t, T]}, \forall \tau \in[t, T], \phi(\tau, t, \mathbf{x}, \mathbf{u}(\cdot)) \in K\right\} \\
\mathcal{I}(t, K) & =\left\{\mathbf{x} \in \mathbb{R}^{n} \mid \forall \mathbf{u} \in \mathcal{U}_{[t, T]}, \forall \tau \in[t, T], \phi(\tau, t, \mathbf{x}, \mathbf{u}(\cdot)) \in K\right\} \\
\mathcal{R}(t, K) & =\left\{\mathbf{x} \in \mathbb{R}^{n} \mid \exists \mathbf{u} \in \mathcal{U}_{[t, T]}, \exists \tau \in[t, T], \phi(\tau, t, \mathbf{x}, \mathbf{u}(\cdot)) \in K\right\}
\end{aligned}
$$

By comparing the sets, it can be stated that:

$$
\mathcal{I}(t, K) \subset \mathcal{V}(t, K) \subset \mathcal{R}(t, K)
$$

The principle of duality results in the relationship, as illustrated in Fig. 3,

$$
\mathcal{R}(t, K)=\left(\mathcal{I}\left(t, K^{c}\right)\right)^{c}
$$
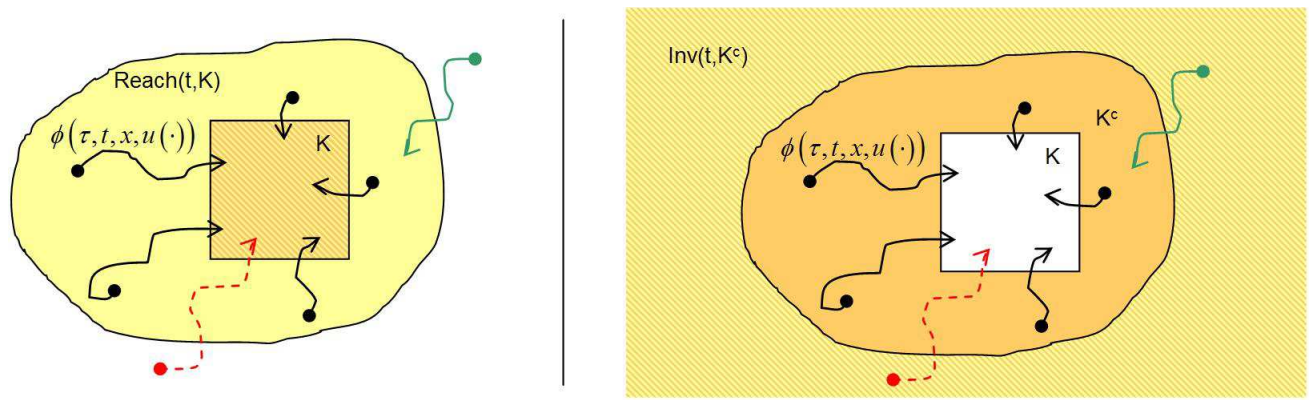

Figure 3. Illustration of the principle of duality. Left $\mathcal{R}(t, K)$, right $\mathcal{I}\left(t, K^{c}\right)$.

The above set characterizations can be rewritten as optimization problems. First a connection is established between viability and the SUPMIN optimal control problem. Assume that the set $K$ is related to the zero level set of a continuous function $l: \mathbb{R}^{n} \rightarrow \mathbb{R}$ by $K=\left\{\mathbf{x} \in \mathbb{R}^{n} \mid l(\mathbf{x})>0\right\}$. Then, it can be stated that:

$$
\mathcal{V}(t, K)=\left\{\mathbf{x} \in \mathbb{R}^{n} \mid V_{1}(\mathbf{x}, t)=\sup _{\mathbf{u}(\cdot) \in \mathcal{U}_{[t, T]}} \min _{\tau \in[t, T]} l(\phi(\tau, t, \mathbf{x}, \mathbf{u}(\cdot)))>0\right\}
$$


The connection between invariance and the INFMIN optimal control problem can be established by considering a closed set $L$, that can be written as the level set of a continuous function $l: \mathbb{R}^{n} \rightarrow \mathbb{R}$, i.e. $L=\left\{\mathbf{x} \in \mathbf{R}^{n} \mid l(\mathbf{x}) \geq 0\right\}$. Then, it can be stated that:

$$
\mathcal{I}(t, L)=\left\{\mathbf{x} \in \mathbb{R}^{n} \mid V_{2}(\mathbf{x}, t)=\inf _{\mathbf{u}(\cdot) \in \mathcal{U}[t, T]} \min _{\tau \in[t, T]} l(\phi(\tau, t, \mathbf{x}, \mathbf{u}(\cdot))) \geqslant 0\right\}
$$

These optimization problems have been transformed in Hamilton-Jacobi-Bellman Partial Differential Equations (HJB PDE) $\stackrel{14}{[1}$ Viability can be written as follows:

$$
\frac{\partial V_{1}}{\partial t}(\mathbf{x}, t)+\min _{\tau \in[t, T]}\left\{\sup _{\mathbf{u}(\cdot) \in \mathcal{U}_{[t, T]}} \frac{\partial V_{1}}{\partial \mathbf{x}}(\mathbf{x}, t) \mathbf{f}(\mathbf{x}, \mathbf{u})\right\}=0
$$

Furthermore, for backward integration $V_{1}(\mathbf{x}, T)=l(\mathbf{x})$ holds. Forward integration implies $V_{1}(\mathbf{x}, t)=l(\mathbf{x})$. Simlarly, invariance is represented by the following HJB PDE:

$$
\frac{\partial V_{2}}{\partial t}(\mathbf{x}, t)+\min _{\tau \in[t, T]}\left\{\inf _{\mathbf{u}(\cdot) \in \mathcal{U}_{[t, T]}} \frac{\partial V_{2}}{\partial \mathbf{x}}(\mathbf{x}, t) \mathbf{f}(\mathbf{x}, \mathbf{u})\right\}=0
$$

In addition, $V_{2}(\mathbf{x}, T)=l(\mathbf{x})$ holds for backward integration, while $V_{2}(\mathbf{x}, t)=l(\mathbf{x})$ applies in case of forward integration. These HJB PDE's can be solved by level sets, for which a toolbox is available in Matlab ${ }^{\circledR 15}$

\section{Approach}

The approach to calculate the safe maneuvering envelope is based on the following steps:

- Define reference boundaries for $V, \gamma$ and $\varphi$ as well as grid step size $\Delta V, \Delta \gamma$ and $\Delta \varphi$

- Define an implicit function accordingly over $V$ and $\gamma$. This needs to be done for every value of $\varphi$ in case speed and flight path angle are bank angle dependent, i.e. $V=V(\varphi)$ and $\gamma=\gamma(\varphi)$.

- The Level sets method toolbox ${ }^{\sqrt{15}}$ relies on the Hamiltonians in Eq. (7) and (8) as a gradient to evolve the implicit function and thus reference boundaries over time

- The cost functions $V_{1}$ and $V_{2}$ become three dimensional functions, where cross sections reflect the situation for a specific time instant $t_{a}$.

- A dissipation function is needed to guarantee numerical stability during these calculations. As a consequence, slightly conservative results are obtained for the boundaries, but analysis has shown that this dissipation has a minor effect on the results. In this specific context, the chosen dissipation function is a Lax Friedrichs dissipation function. 15

\section{Application example}

To illustrate how the envelope estimating algorithm works, a nonlinear 3D aircraft example is considered. At this point, only the slow dynamics as specified in Fig. 2 are considered. Future work will extend to the faster dynamics. The data used in this example are based on the RCAM (Research Civil Aircraft Model) simulation model ${ }^{30}$ The acting forces on the aircraft are illustrated in Fig. 4 for a symmetric flight condition.

For the complete 3D situation, the equations of motion are written as follows: ${ }^{2}$

$$
\begin{aligned}
F_{A_{X}}-W \sin \gamma & =m \dot{V} \\
F_{A_{Z}} \cos \varphi+F_{A_{Y}} \sin \varphi+W \cos \gamma & =-m V \dot{\gamma} \\
-F_{A_{Z}} \sin \varphi+F_{A_{Y}} \cos \varphi & =m V \dot{\chi} \cos \gamma
\end{aligned}
$$

Where the aerodynamic forces can be simplified assuming small aerodynamic angles $\alpha$ and $\beta$ :

$$
\begin{aligned}
& F_{A_{X}}=T \cos \beta \cos \alpha-D(V, \alpha) \approx T-D(V, \alpha) \\
& F_{A_{Z}}=-T \sin \alpha-L(V, \alpha) \approx-L(V, \alpha) \\
& F_{A_{Y}}=-T \sin \beta \cos \alpha+Y_{\text {aero }}(V, \beta) \approx Y_{\text {aero }}(V, \beta)
\end{aligned}
$$




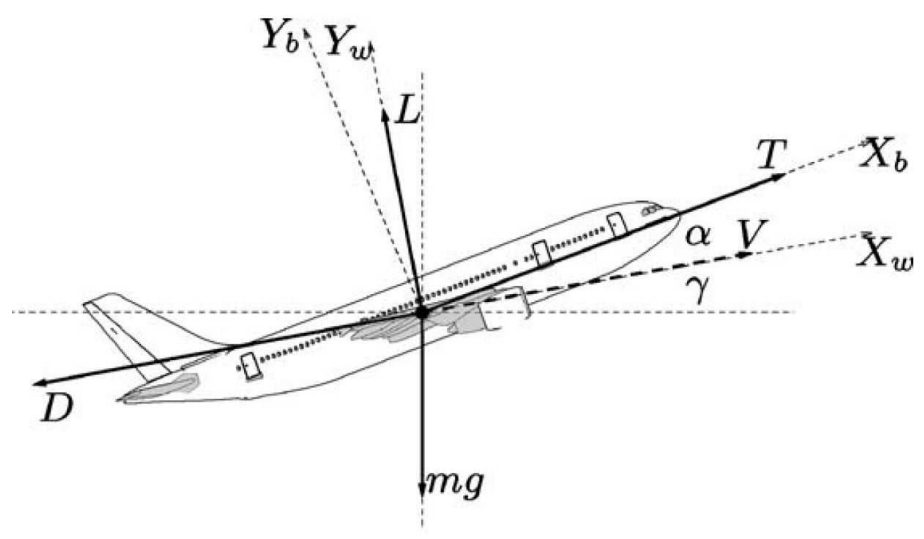

Figure 4. Acting forces on the aircraft model, source: Lygeros 14

with the following expansions for lift $L$, drag $D$ and sideforce $Y_{\text {aero: }}$ :

$$
\begin{aligned}
D(V, \alpha) & =\bar{q} S\left(C_{D_{0}}+C_{D_{\alpha}} \alpha+C_{D_{\alpha^{2}}} \alpha^{2}\right) \\
L(V, \alpha) & =\bar{q} S\left(C_{L_{0}}+C_{L_{\alpha}} \alpha\right) \\
Y_{\text {aero }}(V, \beta) & =\bar{q} S\left(C_{Y_{\beta}} \beta\right)
\end{aligned}
$$

where the dynamic pressure $\bar{q}=1 / 2 \rho V^{2}$.

The corresponding numerical values are: $m=120 \cdot 10^{3} \mathrm{~kg}, g=9.81 \mathrm{~m} / \mathrm{s}^{2}, W=m g, \rho=1.225 \mathrm{~kg} / \mathrm{m}^{3}$ (sea level), $S=260 m^{2}, C_{L_{0}}=1.0656, C_{L_{\alpha}}=6.0723, C_{D_{0}}=0.1599, C_{D_{\alpha}}=0.5035, C_{D_{\alpha^{2}}}=2.1175, C_{Y_{\beta}}=-1.6$.

In the perspective of reachability from stable and controllable trim conditions, the primary states of interest are airspeed $V$ and flight path angle $\gamma$. Considering time scale separation as presented in Fig. 22, the virtual inputs for the slow dynamics are roll angle $\varphi$, angle of attack $\alpha$, sideslip angle $\beta$ and thrust $T$. This framework and combining Eqs. (9) -(17) allows to define the general dynamics $\mathbf{f}$ in Eq. (1) by the following differential equation:

$$
\begin{array}{r}
{\left[\begin{array}{c}
\dot{V} \\
\dot{\gamma}
\end{array}\right]=\left[\begin{array}{c}
-\frac{\rho S}{2 m} V^{2} C_{D_{0}}-g \sin \gamma \\
-\frac{g}{V} \cos \gamma
\end{array}\right]+\left[\begin{array}{c}
\cos \alpha \cos \beta \\
(\cos \varphi \sin \alpha \cos \beta-\sin \varphi \sin \beta) \frac{1}{V}
\end{array}\right] \frac{T}{m}+} \\
+\left[\begin{array}{c}
-\frac{\rho S}{2 m} V^{2}\left(C_{D_{\alpha}} \alpha+C_{D_{\alpha^{2}}} \alpha^{2}\right) \\
\frac{\rho S}{2 m} V\left(C_{L_{0}}+C_{L_{\alpha}} \alpha\right) \cos \varphi
\end{array}\right]+\left[\begin{array}{c}
0 \\
-\frac{\rho S}{2 m} V C_{Y_{\beta}} \beta \sin \varphi
\end{array}\right]
\end{array}
$$

Assuming small aerodynamic angles, as earlier, simplifies the differential equation:

$$
\left[\begin{array}{c}
\dot{V} \\
\dot{\gamma}
\end{array}\right]=\left[\begin{array}{c}
-\frac{\rho S}{2 m} V^{2} C_{D_{0}}-g \sin \gamma \\
-\frac{g}{V} \cos \gamma
\end{array}\right]+\left[\begin{array}{c}
\frac{1}{m} \\
0
\end{array}\right] T+\left[\begin{array}{c}
-\frac{\rho S}{2 m} V^{2}\left(C_{D_{\alpha}} \alpha+C_{D_{\alpha^{2}}} \alpha^{2}\right) \\
\frac{\rho S}{2 m} V\left(C_{L_{0}}+C_{L_{\alpha}} \alpha\right) \cos \varphi
\end{array}\right]+\left[\begin{array}{c}
0 \\
-\frac{\rho S}{2 m} V C_{Y_{\beta}} \beta \sin \varphi
\end{array}\right]
$$

This results in a Hamiltonian function with decoupled virtual inputs $T, \alpha$ and $\beta$. Roll angle $\varphi$ is not decoupled, but will be treated as a discretely gridded input. This decoupling significantly promotes computational efficiency, which is crucial for on-line applications. The Hamiltonian function becomes:

$$
H(\mathbf{p}, \mathbf{x}, \mathbf{u})=\frac{p_{1}}{m} T-p_{1} \frac{\rho S}{2 m} V^{2} C_{D_{\alpha^{2}}} \alpha^{2}+\frac{\rho S}{2 m} V\left(p_{2} C_{L_{\alpha}} \cos \varphi-p_{1} V C_{D_{\alpha}}\right) \alpha-p_{2} \frac{\rho S}{2 m} V C_{Y_{\beta}} \sin \varphi \beta
$$

where $\mathbf{p}$ are the co-states of the value function: $p_{1}=\frac{\partial V_{1}}{\partial V}$ and $p_{2}=\frac{\partial V_{1}}{\partial \gamma}$. This Hamiltonian is linear in thrust $T$ and sideslip angle $\beta$ and quadratic in angle of attack $\alpha$. This structure allows for an efficient optimization routine over the inputs. The allowed ranges of the virtual inputs are: $T_{\min }=20546 \mathrm{~N}, T_{\max }=410920 \mathrm{~N}$, $\alpha_{\min }=0^{\circ}, \alpha_{\max }=14.5^{\circ}$ (no stall), $\phi_{\min / \max }= \pm 60^{\circ}, \beta_{\min / \max }= \pm 5^{\circ}$. The maximizers $\hat{T}, \hat{\alpha}$ and $\hat{\beta}$ depend on the sign of the costates $p_{1}$ and $p_{2}$. Recall that $V>0, C_{D_{\alpha}}>0, C_{D_{\alpha^{2}}}>0, C_{L_{\alpha}}>0, C_{Y_{\beta}}<0$. Due to the underlying physics, no sign changes for these parameters are to be expected in case of structural changes. 
Define $\hat{p}=\frac{p_{2} C_{L_{\alpha}} \cos \varphi-p_{1} V C_{D_{\alpha}}}{2 p_{1} V C_{\alpha_{\alpha}}}$ and $\bar{\alpha}=\frac{\alpha_{\min }+\alpha_{\max }}{2}$. Then the optimizing control inputs can be defined, according to the purpose.

For invariance, the infmin optimization is achieved by means of the following inputs:

- If $p_{1}>0$ then $\hat{T}=T_{\min }$ and

$$
\begin{aligned}
& \text { if } \hat{p}>\bar{\alpha} \text { then } \hat{\alpha}=\alpha_{\min } \\
& \text { if } \hat{p}=\bar{\alpha} \text { then } \hat{\alpha}=\alpha_{\min } \text { or } \alpha_{\max } \\
& \text { if } \hat{p}<\bar{\alpha} \text { then } \hat{\alpha}=\alpha_{\max }
\end{aligned}
$$

- If $p_{1}=0$ then $\hat{T} \in\left[T_{\min } ; T_{\max }\right]$ and

$$
\begin{aligned}
& \text { if } p_{2}>0 \text { then } \hat{\alpha}=\alpha_{\min } \\
& \text { if } p_{2}=0 \text { then } \hat{\alpha} \in\left[\alpha_{\min } ; \alpha_{\max }\right] \\
& \text { if } p_{2}<0 \text { then } \hat{\alpha}=\alpha_{\max }
\end{aligned}
$$

- If $p_{1}<0$ then $\hat{T}=T_{\max }$ and

$$
\begin{aligned}
& \text { if } \hat{p} \leq \alpha_{\min } \text { then } \hat{\alpha}=\alpha_{\min } \\
& \text { if } \alpha_{\min } \leq \hat{p} \leq \alpha_{\max } \text { then } \hat{\alpha}=\hat{p} \\
& \text { if } \hat{p} \geq \alpha_{\max } \text { then } \hat{\alpha}=\alpha_{\max }
\end{aligned}
$$

Besides, for the sideslip angle $\beta$ holds:

- if $p_{2} \sin \varphi>0$ then $\hat{\beta}=\beta_{\text {min }}$

- if $p_{2} \sin \varphi=0$ then $\hat{\beta} \in\left[\beta_{\min } ; \beta_{\max }\right]$

- if $p_{2} \sin \varphi<0$ then $\hat{\beta}=\beta_{\max }$
Alternative inputs are defined for the supmin optimization related to viability:

- If $p_{1}<0$ then $\hat{T}=T_{\max }$ and

$$
\begin{aligned}
& \text { if } \hat{p}>\bar{\alpha} \text { then } \hat{\alpha}=\alpha_{\min } \\
& \text { if } \hat{p}=\bar{\alpha} \text { then } \hat{\alpha}=\alpha_{\min } \text { or } \alpha_{\max } \\
& \text { if } \hat{p}<\bar{\alpha} \text { then } \hat{\alpha}=\alpha_{\max }
\end{aligned}
$$

- If $p_{1}=0$ then $\hat{T} \in\left[T_{\min } ; T_{\max }\right]$ and

$$
\begin{aligned}
& \text { if } p_{2}<0 \text { then } \hat{\alpha}=\alpha_{\min } \\
& \text { if } p_{2}=0 \text { then } \hat{\alpha} \in\left[\alpha_{\min } ; \alpha_{\max }\right] \\
& \text { if } p_{2}>0 \text { then } \hat{\alpha}=\alpha_{\max }
\end{aligned}
$$

- If $p_{1}>0$ then $\hat{T}=T_{\min }$ and

$$
\begin{aligned}
& \text { if } \hat{p} \leq \alpha_{\min } \text { then } \hat{\alpha}=\alpha_{\min } \\
& \text { if } \alpha_{\min } \leq \hat{p} \leq \alpha_{\max } \text { then } \hat{\alpha}=\hat{p} \\
& \text { if } \hat{p} \geq \alpha_{\max } \text { then } \hat{\alpha}=\alpha_{\max }
\end{aligned}
$$

Besides, for the sideslip angle $\beta$ holds:

- if $p_{2} \sin \varphi<0$ then $\hat{\beta}=\beta_{\min }$

- if $p_{2} \sin \varphi=0$ then $\hat{\beta} \in\left[\beta_{\min } ; \beta_{\max }\right]$

- if $p_{2} \sin \varphi>0$ then $\hat{\beta}=\beta_{\max }$

For the purpose of reachability analysis, the principle of duality can be applied for the infmin optimization inputs, as highlighted in Eq. (4).

\section{Results}

The first set of results focuses on motion in the plane of symmetry, assuming bank angle $\varphi=0$. Thereafter, motion leaving the plane of symmetry will be considered.

Fig. 5 illustrates the definition of invariance, viability and reachability in the context of the RCAM example. All these properties are considered in the backward sense, meaning timesteps are taken backward in time. As a reference, the safe trim envelope $K$ is defined as the area between the boundaries $V_{\min }=$ $60 \mathrm{~m} / \mathrm{s}, V_{\max }=100 \mathrm{~m} / \mathrm{s}, \gamma_{\min }=-10^{\circ}$ and $\gamma_{\max }=+10^{\circ}$ and is described by the implicit function $: l(\mathbf{x})=$ $\min \left\{x_{1}-V_{\min }, V_{\max }-x_{1}, x_{2}-\gamma_{\min }, \gamma_{\max }-x_{2}\right\}$ such that $l(\mathbf{x}) \geq 0$ for $\mathbf{x} \in K$ and $l(\mathbf{x})<0$ for $\mathbf{x} \notin K$. In Fig. 5(a), the green area shows the invariant set $\mathcal{I}(T=2 s, K)$ as defined in Sec. II Monte Carlo analyses confirm the accuracy of the result. For initial conditions $\mathbf{x}_{0}$ inside the invariant set $\mathcal{I}(T=2 s, K)$, the whole state trajectory $\phi(\tau, t, \mathbf{x}, \mathbf{u})$ till the end point at $T=2 s$ will remain inside the reference envelope $K$, independent from which inputs $\mathbf{u}(\cdot)$ are applied to the aircraft. For initial conditions $\mathbf{x}_{0}$ outside the invariant set $\mathcal{I}(T=2 s, K)$, there exists at least one input $\mathbf{u}(\cdot)$ which will push part of the state trajectory $\phi(\tau, t, \mathbf{x}, \mathbf{u})$ towards the end point at $T=2 s$ outside the trim envelope $K$. This complies with the aforementioned definition of invariant sets in Section II

As can be seen in Fig. 5(b) the viability set $\mathcal{V}(T=2 s, K)$ is somewhat less restrictive. Monte Carlo analyses confirm that, for initial conditions $\mathbf{x}_{0}$ inside the viability set $\mathcal{V}(T=2 s, K)$, it is always possible to find at least one admissible input $\mathbf{u}(\cdot)$ within saturation bounds which will keep the whole state trajectory $\phi(\tau, t, \mathbf{x}, \mathbf{u})$ till the end point at $T=2 s$ inside the reference envelope $K$. On the other hand, outside the viability set $\mathcal{V}(T=2 s, K)$, the state trajectory $\phi(\tau, t, \mathbf{x}, \mathbf{u})$ is guaranteed to leave the reference envelope $K$ within $T=2 s$, independent from which input $\mathbf{u}$ is applied.

Finally, the concept of backwards reachability $\mathcal{R}(T=2 s, K)$ is illustrated in Fig. 5(c) For initial 
conditions $\mathbf{x}_{0}$ within the backwards reachable set $\mathcal{R}(T=2 s, K)$, it is always possible to find at least one admissible input $\mathbf{u}(\cdot)$ which will bring part of the state trajectory $\phi(\tau, t, \mathbf{x}, \mathbf{u})$ towards the end point at $T=2 s$ inside the trim envelope $K$. On the other hand, from outside the backwards reachability set $\mathcal{R}(T=2 s, K)$, it is impossible for the state trajectory $\phi(\tau, t, \mathbf{x}, \mathbf{u})$ to reach the reference envelope $K$ within $T=2 s$, independent from which input $\mathbf{u}$ is applied.

Fig. 6] shows the backwards evolution of invariance and viability sets over different discrete time steps of a total backwards time horizon $T=7 \mathrm{~s}$. At $t=7 \mathrm{~s}$, both sets are identical to the reference envelope $K$. Over the next few seconds, both sets start shrinking. While the invariant set keeps shrinking and eventually disappears altogether around $t=4.6 \mathrm{~s}$, the viability set converges to a steady state situation, which is reached around $t=3 \mathrm{~s}$. The difference between both can be analysed from their definition. For a time horizon long enough (in this specific case 4.6s), it is always possible to find at least one input within saturation limits which will push the state trajectory starting at whatever initial condition outside the reference envelope. The converging viability set towards a steady state shape indicates that there is a critical time instant (in this specific case 3s) for which at least one input can be found which keeps the state trajectories from the viable initial conditions within the reference envelope. Prolonging the time horizon won't shrink this viability set further. This observation allows to make a time independent statement: the viability set for $t=3 \mathrm{~s}$ and less is the set of initial conditions for which at least one admissible input can be found to keep the state trajectory within the reference envelope, irrespective of the length of the time horizon. Outside this viability set, the state trajectory is guaranteed to leave the reference envelope eventually at some point, independent from

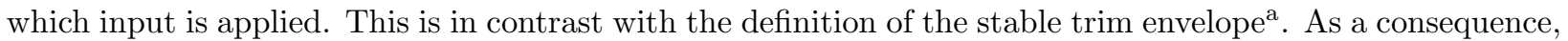
it can be stated that under any circumstance the stable trim envelope should be situated inside the viability set.

The backwards invariance, viability and reachability sets for the RCAM model over a time horizon of $T=2 s$ are compared in Fig. 7 . This set comparison confirms that invariance is a subset of viability, which in its turn is a subset of reachability, as was already claimed in Eq. (3).

So far, only backwards integration has been considered. Besides, forward integration is important in the interpretation of the safe maneuvering envelope as elaborated in Section I The only difference between both is the direction of the time horizon and the time steps: backwards reachability considers rearward timesteps, as has been discussed so far in this section, while forwards reachability involves forward time steps. Fig. 8 compares forward and backward reachability for the RCAM model over a time horizon $T=2 s$. Backwards reachability is the lower set and is consistent with previous figures, forwards reachability is the upper envelope. According to the definition of the safe maneuvering envelope provided in Section \ the cross section between both in the center is designated as the safe maneuvering envelope. All flight conditions in this set can be reached from the stable trim envelope, and the stable trim envelope can be reached from this set as well.

Despite the transparent definition of the safe maneuvering envelope provided previously, this set of flight conditions is somewhat too restrictive in the context of fault tolerant control and upset recovery. For this purpose, the backwards reachable set can be defined as the survivable envelope. This envelope corresponds to the set of flight conditions from which a safe recovery to a stable trim flight condition is still possible within the specified time horizon $T$ after a disturbing failure, wind gust or other upset. As an illustration of this concept, the safe and survivable envelopes for the RCAM model over a time horizon $T=2 s$ are given in Fig. 9 (dark and light green respectively), together with the reference envelope $K$ which is marked by the red contour. The pilot can safely steer the aircraft arbitrarily as desired inside the dark green safe maneuvering envelope within 2s. In case of a failure, wind gust or other upset, it is possible to recover towards a safe equilibrium condition within 2 s as long as the initial condition immediately after perturbation is situated inside the light green survivable envelope. However, given the admissible inputs between saturation limits, it is impossible for the pilot to steer the plane actively towards the light green area. In the next examples, focus will be placed on the survivable maneuvering envelope.

Some damage scenarios are considered in Fig. 10, and their influence on the survivable maneuvering

${ }^{\text {a }}$ The trim envelope is the set of flight conditions for which an equilibrium can be reached, meaning that an admissible input within saturation limits can be found for these flight conditions which renders all values of the first order time derivative of the state vector equal to zero. Stability can be defined as static and dynamic stability. An equilibrium point is statically stable when a deviation from equilibrium, caused by a disturbance, will automatically generate a force or moment in the opposite direction with respect to the deviation. A dynamically stable trim point is a statically stable equilibrium point where the force or moment, automatically generated by the disturbance, is large enough to overcome and eliminate the disturbance. In this paper, a stable trim envelope is de facto the collection of flight conditions in a dynamically stable equilibrium. 

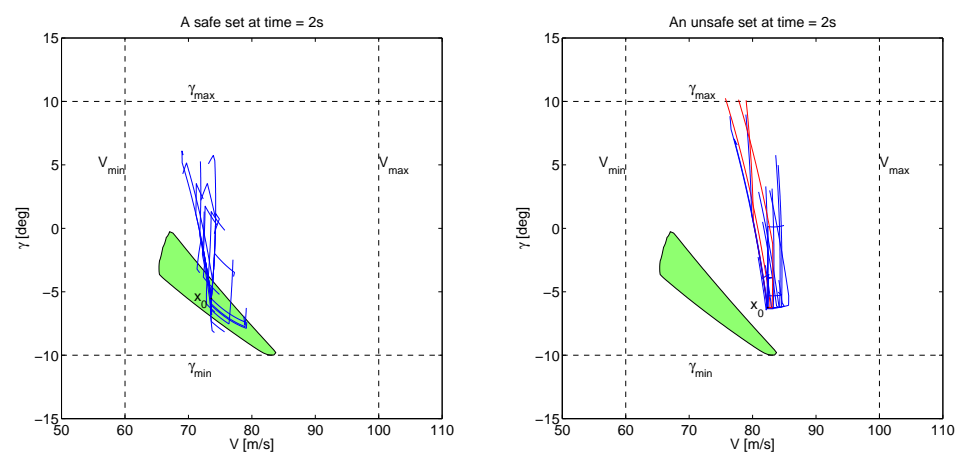

(a) Backwards invariance with Monte Carlo Analysis
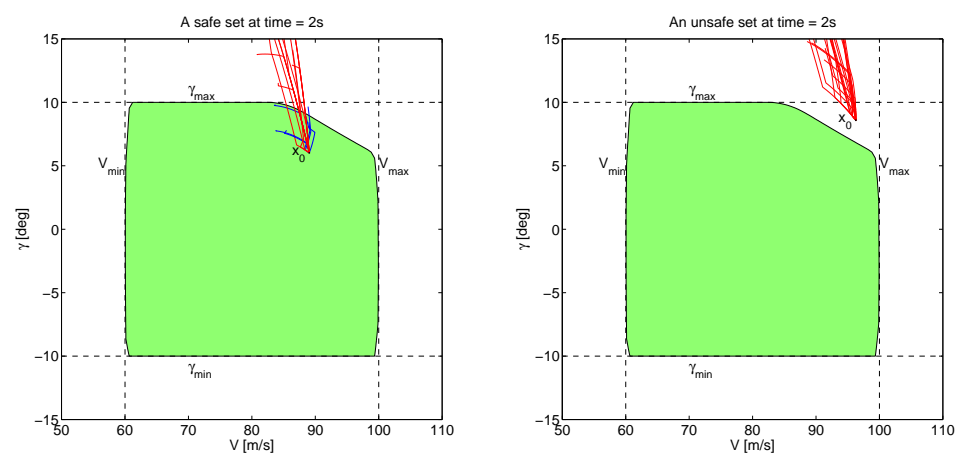

(b) Backwards viability with Monte Carlo Analysis
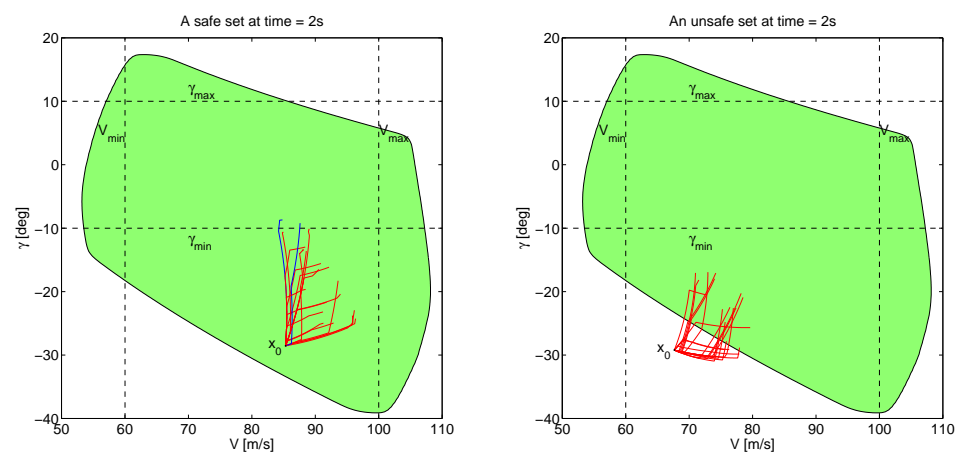

(c) Backwards reachability with Monte Carlo Analysis

Figure 5. Backwards invariance, viability and reachability for RCAM model for time horizon $T=2 s$, including Monte Carlo Analysis 

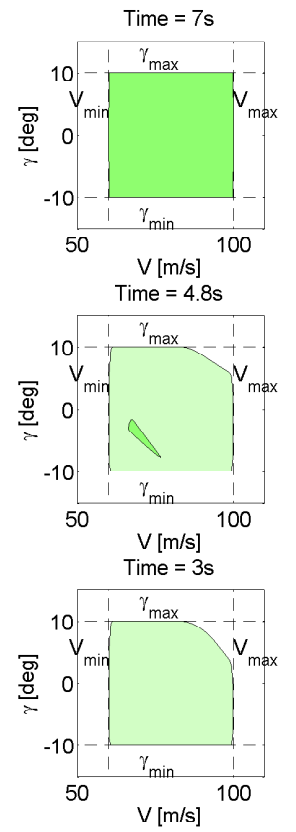
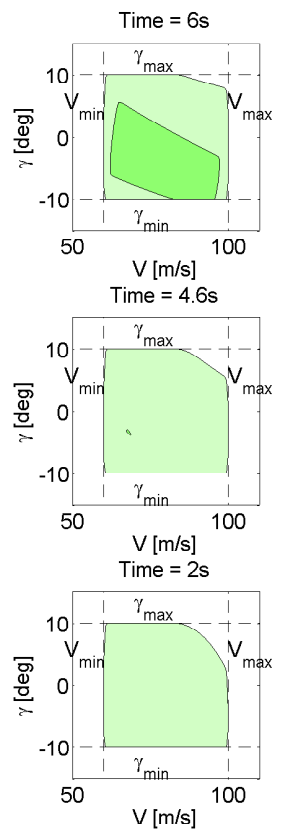
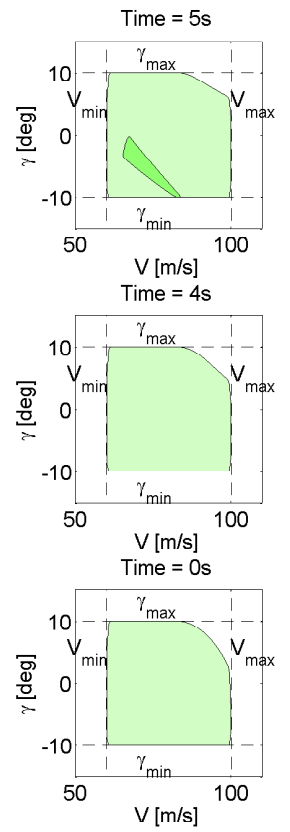

Figure 6. Comparison of backwards invariance and viability for RCAM model over different time instants

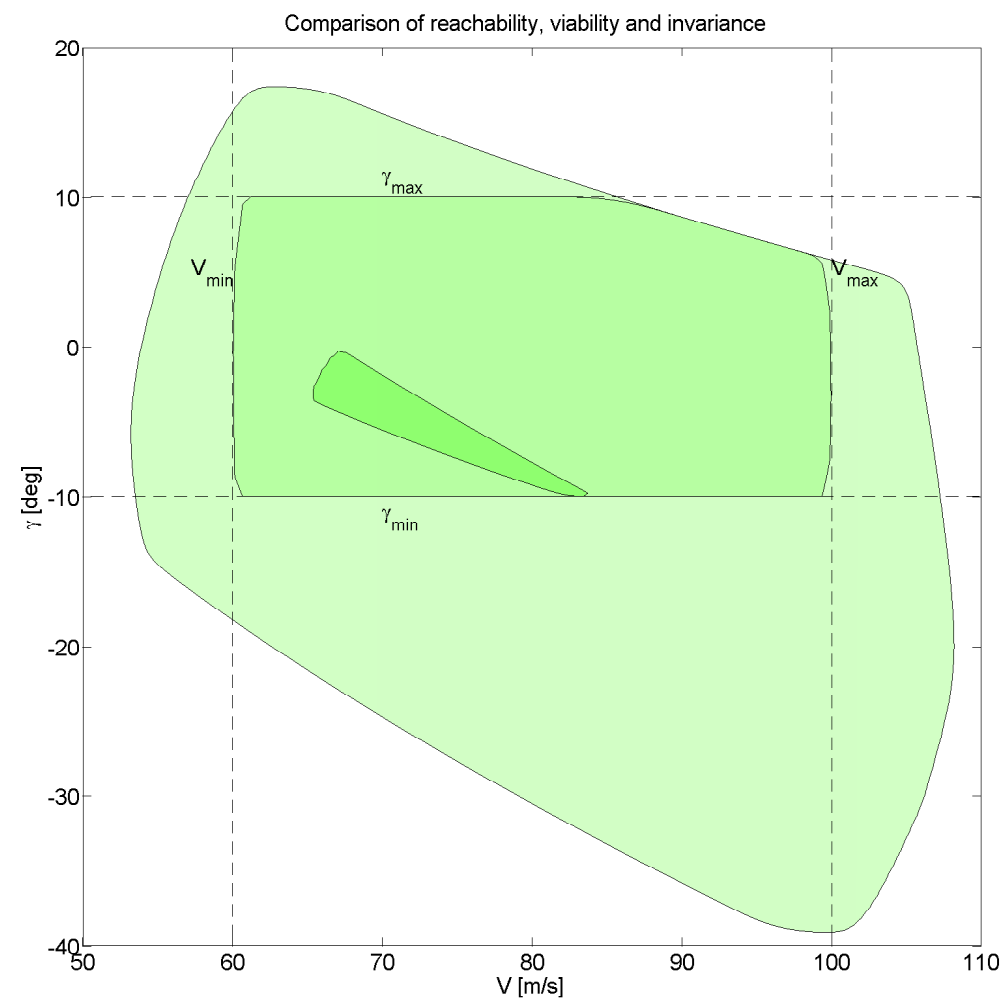

Figure 7. Comparison of backwards invariance, viability and reachability for RCAM model for time horizon $T=2 s$ 


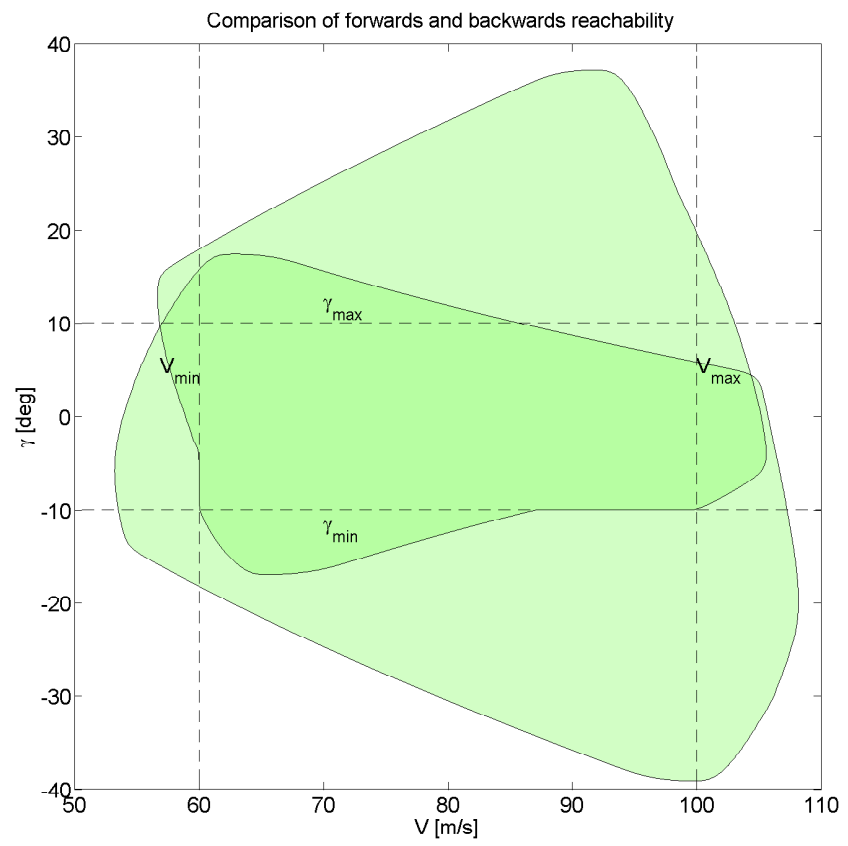

Figure 8. Comparison of forward (upper area) and backward (lower area) reachability for RCAM model for time horizon $T=2 s$

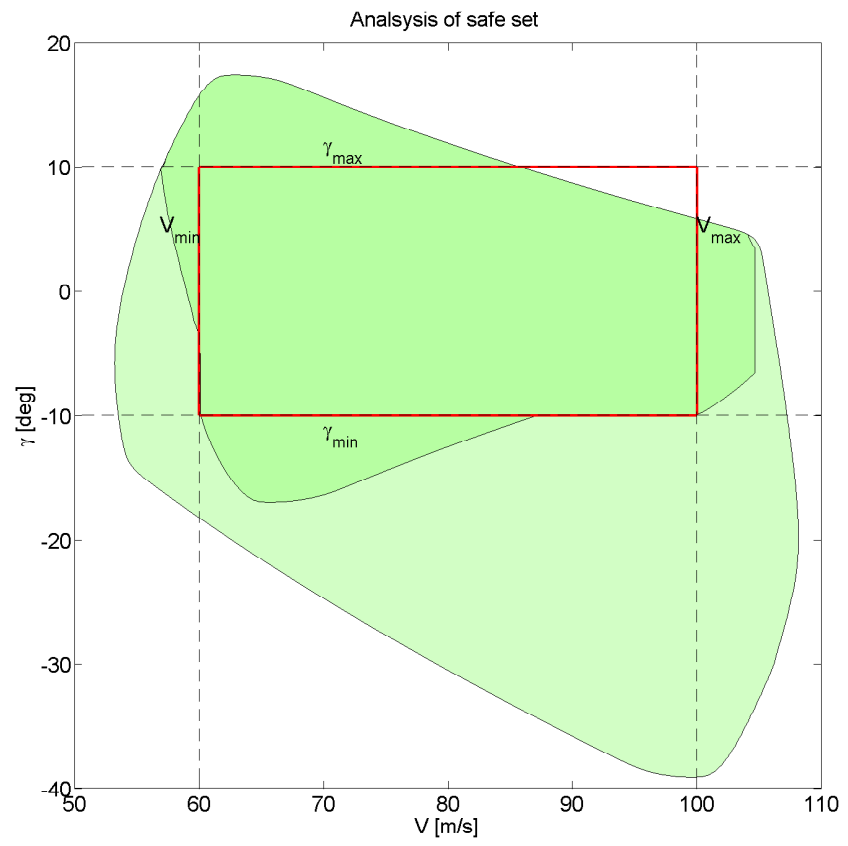

Figure 9. Safe (dark green) and survivable (light green) maneuvering envelopes for RCAM model for time horizon $T=2 s$. 
envelope is investigated. Fig. 10(a) considers a generic damage scenario where the total lift force is reduced by $20 \%$ and the total drag force is increased by $20 \%$. As can be seen in Fig. 10(a), the maneuvering envelope for the damaged aircraft shifts upward and expands slightly to the right. The physical explanation for this is the fact that the loss in lift force results in reduced climb capability but improved diving capability. As a consequence, the reference envelope can be reached from smaller negative, but larger positive flight path angles $\gamma$. Moreover, increased drag leads to a faster deceleration capability, which makes the trim envelope reachable from slightly larger airspeeds within $2 \mathrm{~s}$.

As second damage scenario, an input restriction is defined where engine damage results in a $30 \%$ lower limit of the maximum thrust value. In Fig. 10(b) it can be seen that only the left boundary of the envelope shrinks somewhat. This corresponds to the reduced acceleration capability due to the lower $T_{\max }$. The reference envelope can only be reached from slightly higher speeds in the lower range between $50 \mathrm{~m} / \mathrm{s}$ and $60 \mathrm{~m} / \mathrm{s}$, compared with the nominal situation.

Figure 10(c) shows the cumulative effect of both damage scenarios, where both previous changes and shifts have been combined in the new survivable maneuvering envelope for the RCAM model over a time horizon of $T=2 s$.

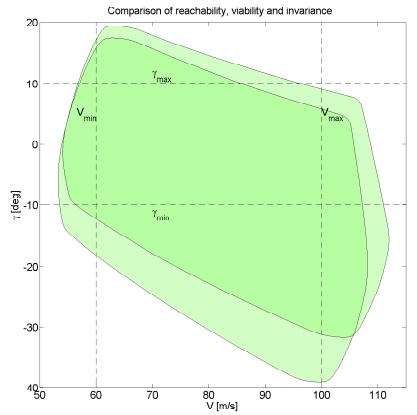

(a) $20 \%$ lift loss and $20 \%$ drag increase

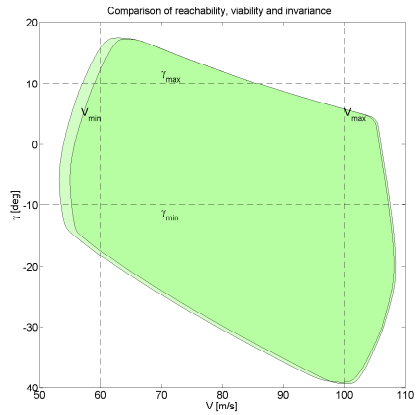

(b) $30 \%$ decrease of $T_{\max }$

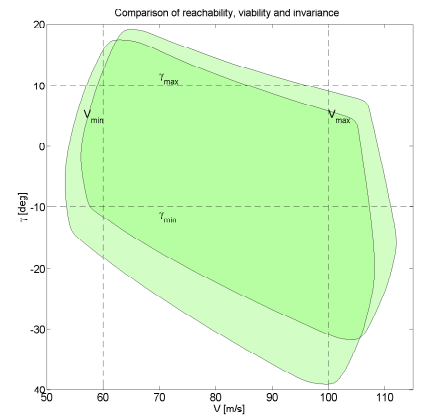

(c) $20 \%$ lift loss and $20 \%$ drag increase as well as $30 \%$ decrease of $T_{\max }$

Figure 10. Different damage scenarios for RCAM model for time horizon $T=2 s$

So far, only motion in the plane of symmetry has been considered. By assuming that bank angle $\varphi \neq 0$, an additional dimension is added to the maneuvering envelope. The computationally most efficient way to do this, is defining a discrete set of bank angle values $\varphi \in\left[0^{\circ} ; 60^{\circ}\right]$, and calculating the $V, \gamma$ maneuvering envelope for every value of $\varphi$ individually. Due to symmetrical properties of the aircraft, only positive bank angles need to be considered. In case of asymmetric damage, the bank angle range needs to be adapted accordingly. The resulting 3D maneuvering envelope is given in Fig. 11. A more extensive analysis of these results will be given in section VI, together with the incorporation of some robustness characteristics in the determination of the survivable maneuvering envelope. 


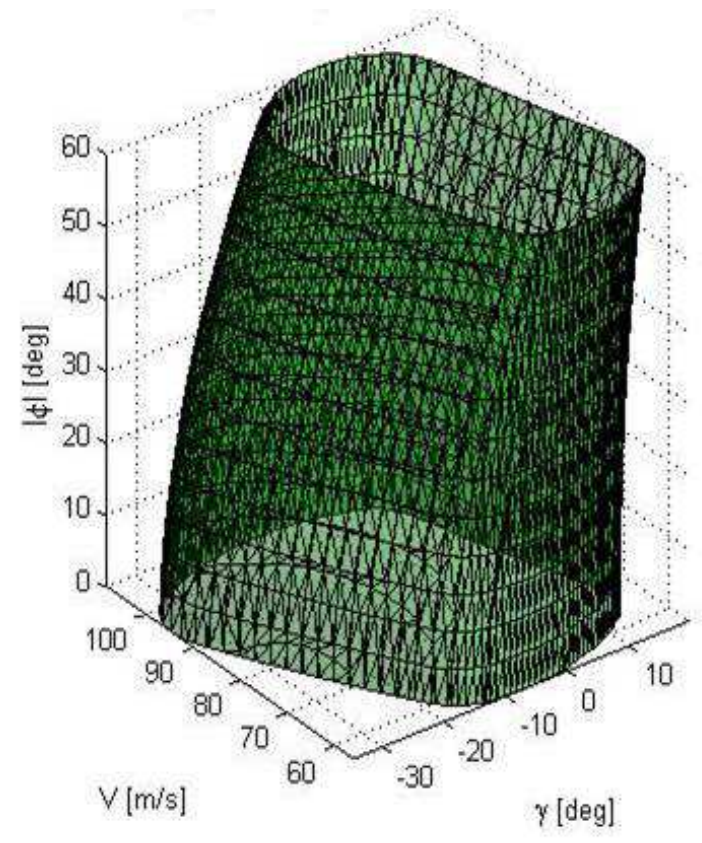

Figure 11. Result of 2D optimization procedure over different bank angles $\phi$, time horizon $T=2 s$

\section{Robustness consideration}

In the optimal control framework elaborated previously, it is possible to incorporate robustness for uncertainties in the estimated values for the aerodynamic derivatives. These uncertainties are based on the standard deviations for the identified aerodynamic parameters:

$$
\begin{aligned}
C_{L_{\bullet}} & =\hat{C}_{L_{\bullet}}+\hat{s}_{L_{\bullet}} \cdot \Delta_{L_{\bullet}} \text { with }\left|\Delta_{L_{\bullet}}\right| \leqslant 1 \\
C_{D_{\bullet}} & =\hat{C}_{D_{\bullet}}+\hat{s}_{D_{\bullet}} \cdot \Delta_{D_{\bullet}} \text { with }\left|\Delta_{D_{\bullet}}\right| \leqslant 1 \\
C_{Y_{\bullet}} & =\hat{C}_{Y_{\bullet}}+\hat{s}_{Y_{\bullet}} \cdot \Delta_{Y_{\bullet}} \text { with }\left|\Delta_{Y_{\bullet}}\right| \leqslant 1
\end{aligned}
$$

Based upon the required degree of conservatism, the standard deviations $\hat{s}_{\bullet}$. can be multiplied by 2 or 3 in order to increase coverage of the Gaussian distribution of the uncertain parameter $\left(1 \hat{s}_{\bullet} .=68 \%, 2 \hat{s}_{\bullet} .=95 \%\right.$, $\left.3 \hat{s}_{\bullet .}=99 \%\right)$.

The crux is to include the $\Delta$ 's as disturbances in the optimization function, they oppose the optimization over $\mathbf{u}$. As a consequence, the invariance optimization formulation becomes: $\frac{19}{19}$

$$
V_{2}(\mathbf{x}, t)=\inf _{\mathbf{u}(\cdot) \in \mathcal{U}[t, T]} \sup _{\boldsymbol{\Delta}(\cdot) \in[-1,1]} \min _{\tau \in[t, T]} l(\phi(\tau, t, \mathbf{x}, \mathbf{u}(\cdot), \boldsymbol{\Delta}(\cdot)))>0
$$

Similarly as previously, reformulation into an HJB PDE yields:

$$
\frac{\partial V_{2}}{\partial t}(\mathbf{x}, t)+\min _{\tau \in[t, T]}\left\{\inf _{\mathbf{u}(\cdot) \in \mathcal{U}_{[t, T]}} \sup _{\boldsymbol{\Delta}(\cdot) \in[-1,1]} \frac{\partial V_{2}}{\partial \mathbf{x}}(\mathbf{x}, t) \mathbf{f}(\mathbf{x}, \mathbf{u}, \boldsymbol{\Delta})\right\}=0
$$

where $V_{2}(\mathbf{x}, T)=l(\mathbf{x})$ holds for backward integration and $V_{2}(\mathbf{x}, t)=l(\mathbf{x})$ applies to forward integration.

For this purpose the Hamiltonian from Eq. (20) can be rewritten, this time including parts independent of the inputs $T, \alpha$ or $\beta$ but with some aerodynamic derivative(s):

$$
\begin{aligned}
H(\mathbf{p}, \mathbf{x}, \mathbf{u})= & -p_{1} \frac{\rho S}{2 m} V^{2} C_{D_{0}}+p_{2} \frac{\rho S}{2 m} V C_{L_{0}} \cos \varphi-p_{1} \frac{\rho S}{2 m} V^{2}\left(C_{D_{\alpha}} \alpha+C_{D_{\alpha^{2}}} \alpha^{2}\right)+p_{2} V \frac{\rho S}{2 m} C_{L_{\alpha}} \cos \varphi \alpha+ \\
& -p_{2} \frac{\rho S}{2 m} V C_{Y_{\beta}} \sin \varphi \beta
\end{aligned}
$$


It can be observed that the aerodynamic derivatives all appear linearly in an uncoupled way, which allows a similar procedure to solve the optimization as previously. By rewriting the Hamiltonian as a summation of terms, where each term is a multiplication of a variable involving a costate, a constant factor and a derivative, one can determine the sign of this factor, which consists of the predefined physical parameters:

$$
\begin{aligned}
H(\mathbf{p}, \mathbf{x}, \mathbf{u})= & -p_{1} \underbrace{\frac{\rho S}{2 m} V^{2}}_{>0} C_{D_{0}}+p_{2} \underbrace{\frac{\rho S}{2 m} V \cos \varphi}_{>0} C_{L_{0}}-p_{1} \underbrace{\frac{\rho S}{2 m} V^{2} \alpha^{2}}_{>0} C_{D_{\alpha^{2}}}+p_{2} \underbrace{\frac{\rho S}{2 m} V \alpha \cos \varphi}_{>0} C_{L_{\alpha}}+ \\
& -p_{1} \underbrace{\frac{\rho S}{2 m} V^{2} \alpha}_{>0} C_{D_{\alpha}}-p_{2} \sin \varphi \beta \underbrace{\frac{\rho S}{2 m} V}_{>0} C_{Y_{\beta}}
\end{aligned}
$$

where it should be noted that $\varphi \in\left[-60^{\circ} ; 60^{\circ}\right], \alpha \in\left[0^{\circ} ; 14.5^{\circ}\right], \beta \in\left[-5^{\circ} ; 5^{\circ}\right]$. Furthermore airspeed $V>0$ and for the aerodynamic derivatives, it is known that $C_{D_{\alpha}}>0, C_{D_{\alpha^{2}}}>0, C_{L_{\alpha}}>0, C_{Y_{\beta}}<0$. Due to the underlying physics, no sign changes for these parameters are to be expected in case of uncertainty or structural changes.

Based upon this formulation, optimal control inputs for the aerodynamic derivatives can be defined as given in Table 1 where $\bar{C}_{\bullet}=C_{\bullet_{\max }}$ and $\underline{C} \cdot C_{\bullet} \bullet_{\min }$.

Table 1. Optimal control inputs for robustness against uncertain aerodynamic derivatives

\begin{tabular}{ccc}
\hline \hline sign of costate & minimizer & maximizer \\
\hline$p_{1} \geq 0$ & $\hat{C}_{D_{0}}=\bar{C}_{D_{0}}, \hat{C}_{D_{\alpha}}=\bar{C}_{D_{\alpha}}, \hat{C}_{D_{\alpha^{2}}}=\bar{C}_{D_{\alpha^{2}}}$ & $\hat{C}_{D_{0}}=\underline{C}_{D_{0}}, \hat{C}_{D_{\alpha}}=\underline{C}_{D_{\alpha}}, \hat{C}_{D_{\alpha^{2}}}=\underline{C}_{D_{\alpha^{2}}}$ \\
$p_{1}<0$ & $\hat{C}_{D_{0}}=\underline{C}_{D_{0}}, \hat{C}_{D_{\alpha}}=\underline{C}_{D_{\alpha}}, \hat{C}_{D_{\alpha^{2}}}=\underline{C}_{D_{\alpha}{ }^{2}}$ & $\hat{C}_{D_{0}}=\bar{C}_{D_{0}}, \hat{C}_{D_{\alpha}}=\bar{C}_{D_{\alpha}}, \hat{C}_{D_{\alpha^{2}}}=\bar{C}_{D_{\alpha^{2}}}$ \\
$p_{2} \geq 0$ & $\hat{C}_{L_{0}}=\underline{C}_{L_{0}}, \hat{C}_{L_{\alpha}}=\underline{C}_{L_{\alpha}}$ & $\hat{C}_{L_{0}}=\bar{C}_{L_{0}}, \hat{C}_{L_{\alpha}}=\bar{C}_{L_{\alpha}}$ \\
$p_{2}<0$ & $\hat{C}_{L_{0}}=\bar{C}_{L_{0}}, \hat{C}_{L_{\alpha}}=\bar{C}_{L_{\alpha}}$ & $\hat{C}_{L_{0}}=\underline{C}_{L_{0}}, \hat{C}_{L_{\alpha}}=\underline{C}_{L_{\alpha}}$ \\
$p_{2} \sin \varphi \cdot \beta \geq 0$ & $\hat{C}_{Y_{\beta}}=\bar{C}_{Y_{\beta}}$ & $\hat{C}_{Y_{\beta}}=\underline{C}_{Y_{\beta}}$ \\
$p_{2} \sin \varphi \cdot \beta<0$ & $\hat{C}_{Y_{\beta}}=\underline{C}_{Y_{\beta}}$ & $\hat{C}_{Y_{\beta}}=\bar{C}_{Y_{\beta}}$ \\
\hline \hline
\end{tabular}

With this information, it is possible to create an entire "uncertainty band" around the envelope, however, here focus will be placed on the "worst-case" minimal size envelope.

Fig. 12 compares the 3D envelopes with and without uncertainty, where two levels of uncertainty have been considered here, namely $10 \%$ and $20 \%$ of uncertainty on all aerodynamic derivatives. For the purpose of this example, identical ratios of standard deviations over nominal values have been defined for all derivatives, but the algorithm is capable to deal with individual standard deviations which can vary between the different aerodynamic derivatives. It can be clearly seen in Fig. 12 that larger degrees of uncertainty result in more significant shrinking of the envelope, since this is a "worst-case" minimal size envelope.

Fig. 13 analyses the $V, \gamma$ maneuvering envelope for different values of bank angle $\varphi$, including robustness for uncertainties of $10 \%$ and 20\%. By comparing Fig. 13(a), 13(b) and 13(c), it can be seen that larger bank angles have an influence on the climb capability of the aircraft. This is due to the physical principle that climb capability of lift force is provided through $L \cos \varphi$, which confirms a smaller decrease for smaller bank angles (up to $\varphi=25^{\circ}$ as shown in Fig. 13(b) but a much more significant change for larger bank angles as can be seen in Fig. 13(c)

The final scenario to be considered, is the $V, \gamma$ maneuvering envelope for the RCAM model for different values of bank angle $\varphi$, with and without generic damage scenario involving $20 \%$ decrease in lift force and increase in drag force. The results are shown in Fig. 14 and 15. Fig. 14 provides a comparison of the 3D envelopes without considering robustness.

Fig. 15 includes robustness for $10 \%$ uncertainty and analyses the $V, \gamma$ maneuvering envelope for three different values of bank angle $\varphi$, namely $0^{\circ}, 25^{\circ}$ and $60^{\circ}$. By comparing Fig. 15(a), 15(b) and 15(c), it can be seen that the damage scenario as well as larger bank angles both have a negative influence on the climb capability of the aircraft. This is again consistent with the underlying physical principles, as explained previously. For motion out of the plane of symmetry, climb capability is provided through $L \cos \varphi$. In this example, this capability is reduced by the non-zero bank angle $\varphi$ as well as by the reduced lift vector $L$, 


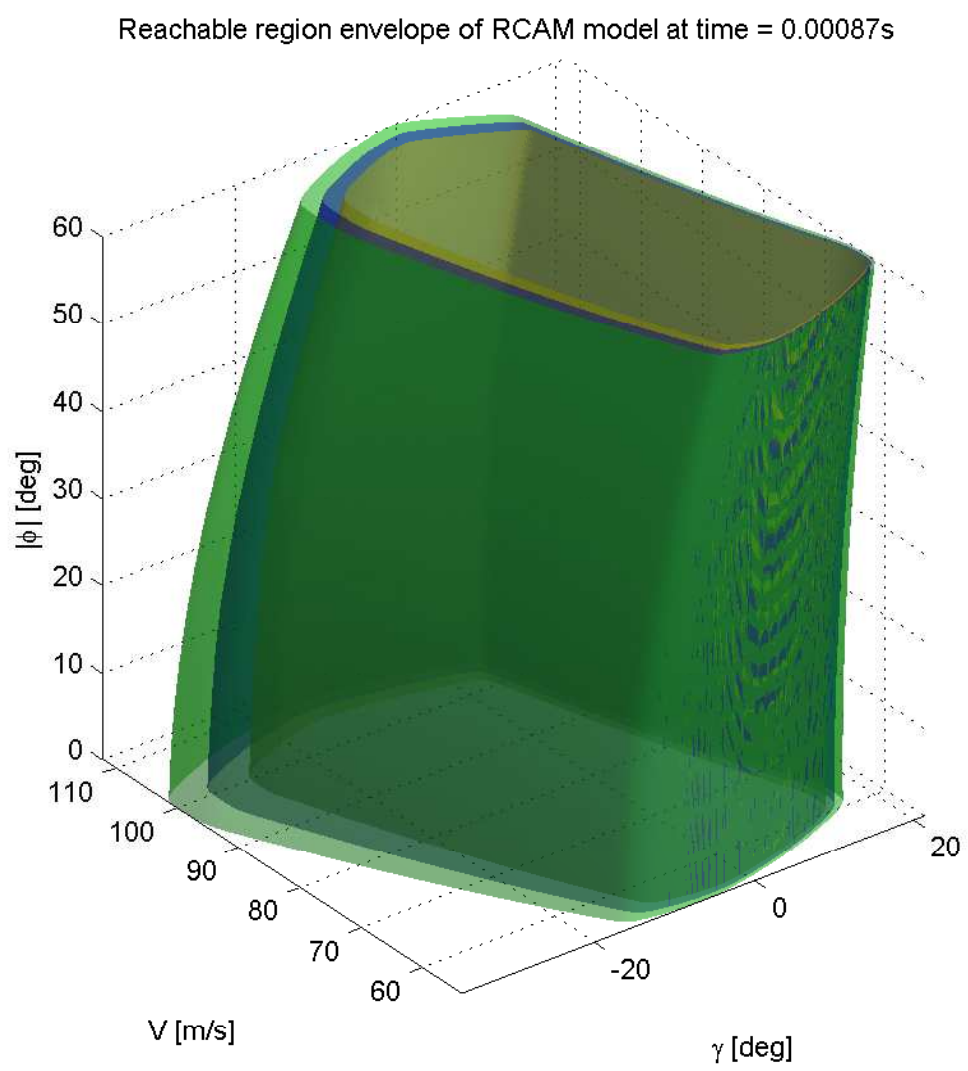

Figure 12. Comparison of $3 \mathrm{D}$ envelopes with and without uncertainty: nominal (green), 10\% uncertainty (blue), 20\% uncertainty (yellow)

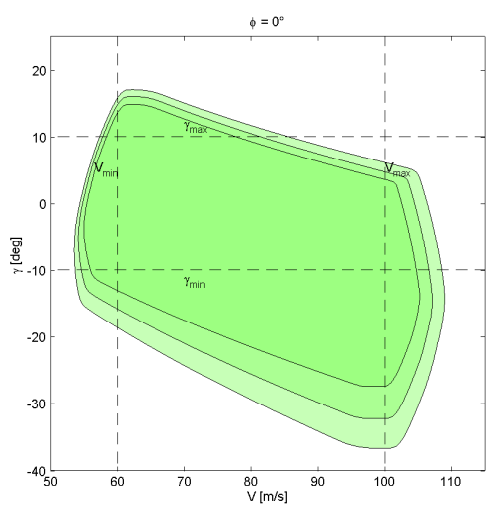

(a) $\varphi=0^{\circ}$

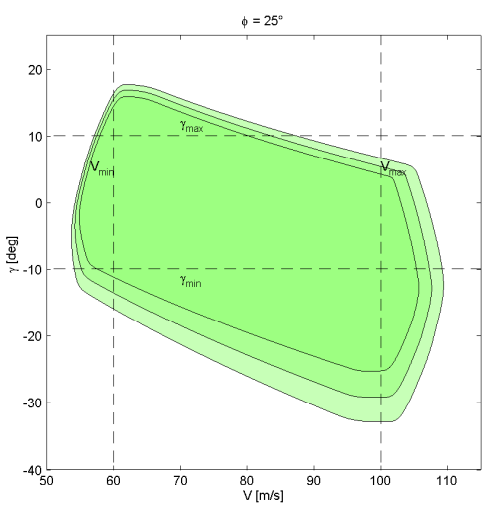

(b) $\varphi=25^{\circ}$

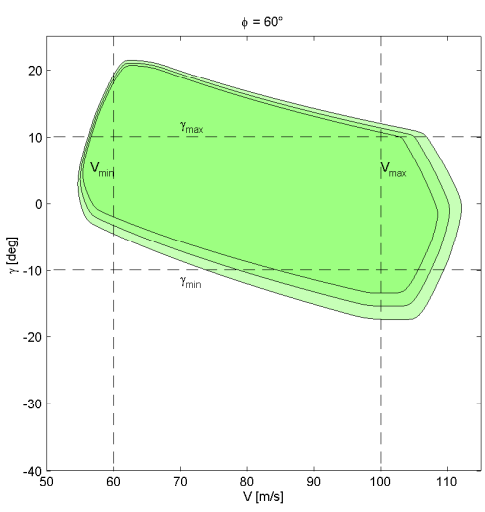

(c) $\varphi=60^{\circ}$

Figure 13. $V, \gamma$ maneuvering envelope of RCAM model for time horizon $T=2 s$ for different bank angles and different uncertainty levels. Smaller envelope areas correspond to larger uncertainty bounds. 


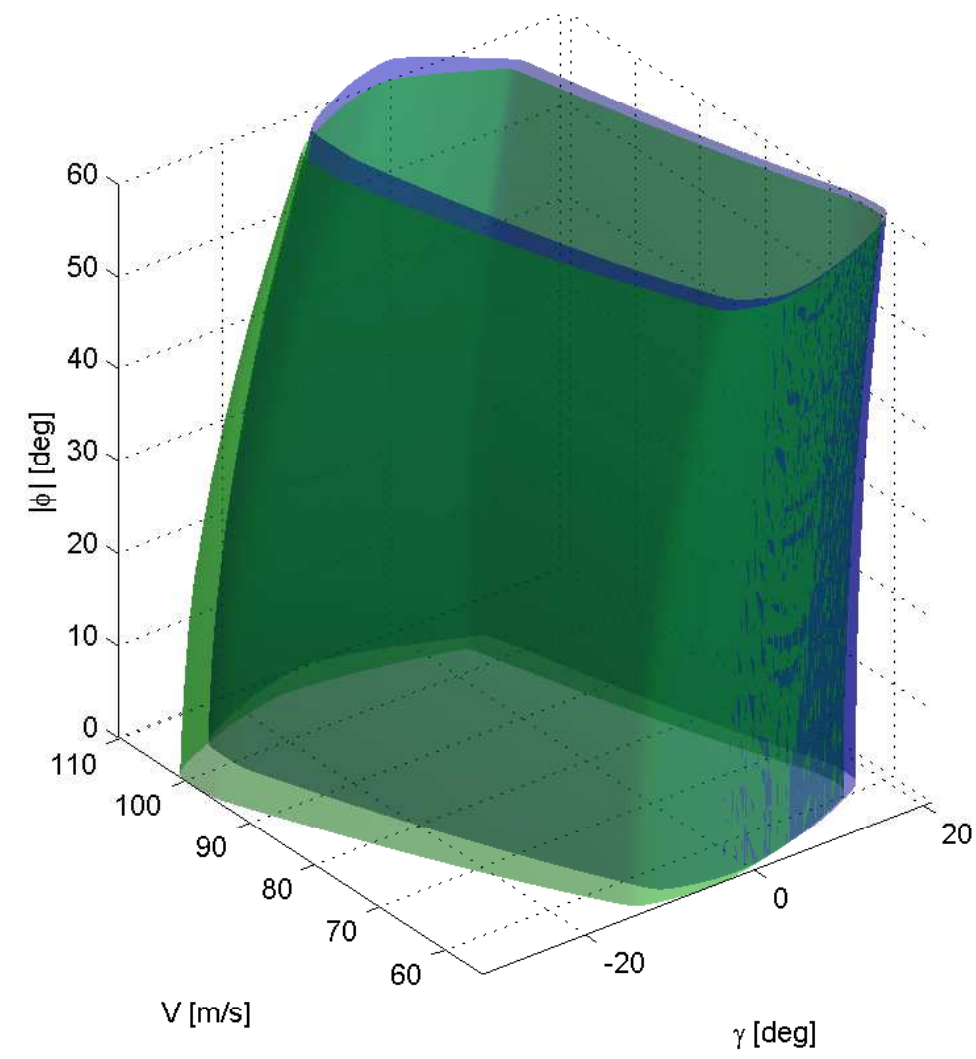

Figure 14. Comparison of $3 \mathrm{D}$ envelopes with and without damage: nominal (blue), $20 \%$ change in lift and drag (green). No uncertainty included.

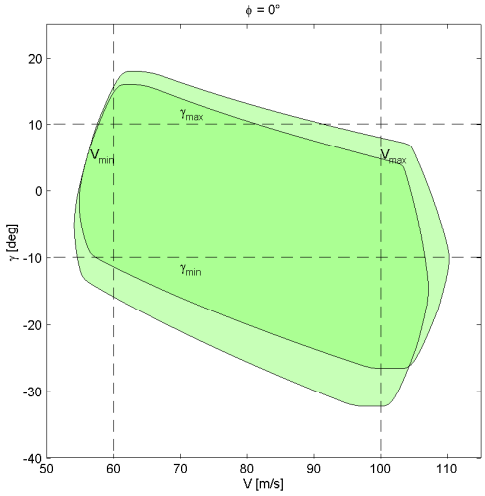

(a) $\varphi=0^{\circ}$

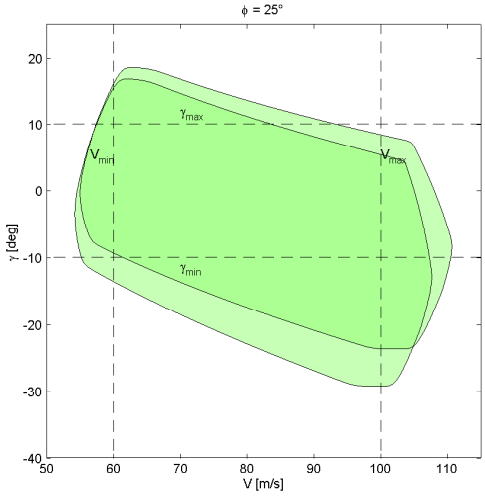

(b) $\varphi=25^{\circ}$

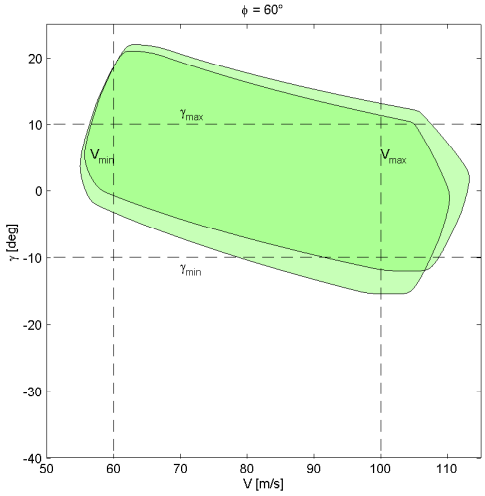

(c) $\varphi=60^{\circ}$

Figure 15. $\quad V, \gamma$ maneuvering envelope of RCAM model without and with $20 \%$ change in lift and drag for time horizon $T=2 s$ for different bank angles. 10\% uncertainty included. Lower envelope areas correspond to nominal configuration. 
resulting in an accumulated effect. This example is the combination of the situations in Fig. 10(a) and 13 , In case of a larger bank angle of $\varphi=60^{\circ}$, the decrease in climb capability due to loss of lift force is less than for zero bank angle, which is confirmed by comparing Fig. 15(a), 15(b) and 15(c).

Extensive Monte Carlo analyses have been performed in order to verify the accuracy of the boundary of the estimated survivable maneuvering envelope. These analyses have been based on the non-simplified aircraft model, ignoring the assumption that the aerodynamic angles $\alpha$ and $\beta$ should be small. All these Monte Carlo analyses have confirmed that the results provided here are accurate and that the simplifications hold for the current ranges of the aerodynamic angles, namely $\alpha \in\left[0^{\circ} ; 14.5^{\circ}\right]$ (no stall) and $\beta \in\left[-5^{\circ} ;+5^{\circ}\right]$. This is an important conclusion which makes a relevant on-line safe maneuvering envelope estimation tool much more feasible.

\section{Information required and output provided to other algorithms}

The research results presented here contribute to the VSST project (Vehicle Safety Systems Technologies) and are planned to be integrated in the task package "Maneuverability Estimation \& Upset Prevention", involving the subtask activities "Dynamic Envelope Estimation \& Protection (DEEP)" and "Constrained Trajectory Generation \& Anticipatory Guidance (CTGAG)".

As a consequence, this maneuvering envelope estimation algorithm is interconnected with other algorithms, namely real time modeling, flight envelope protection, trajectory generation and anticipatory guidance. The first routine provides inputs to the algorithm, while its output is provided to the three latter ones. Figure 16 illustrates these interconnections in a global overview.

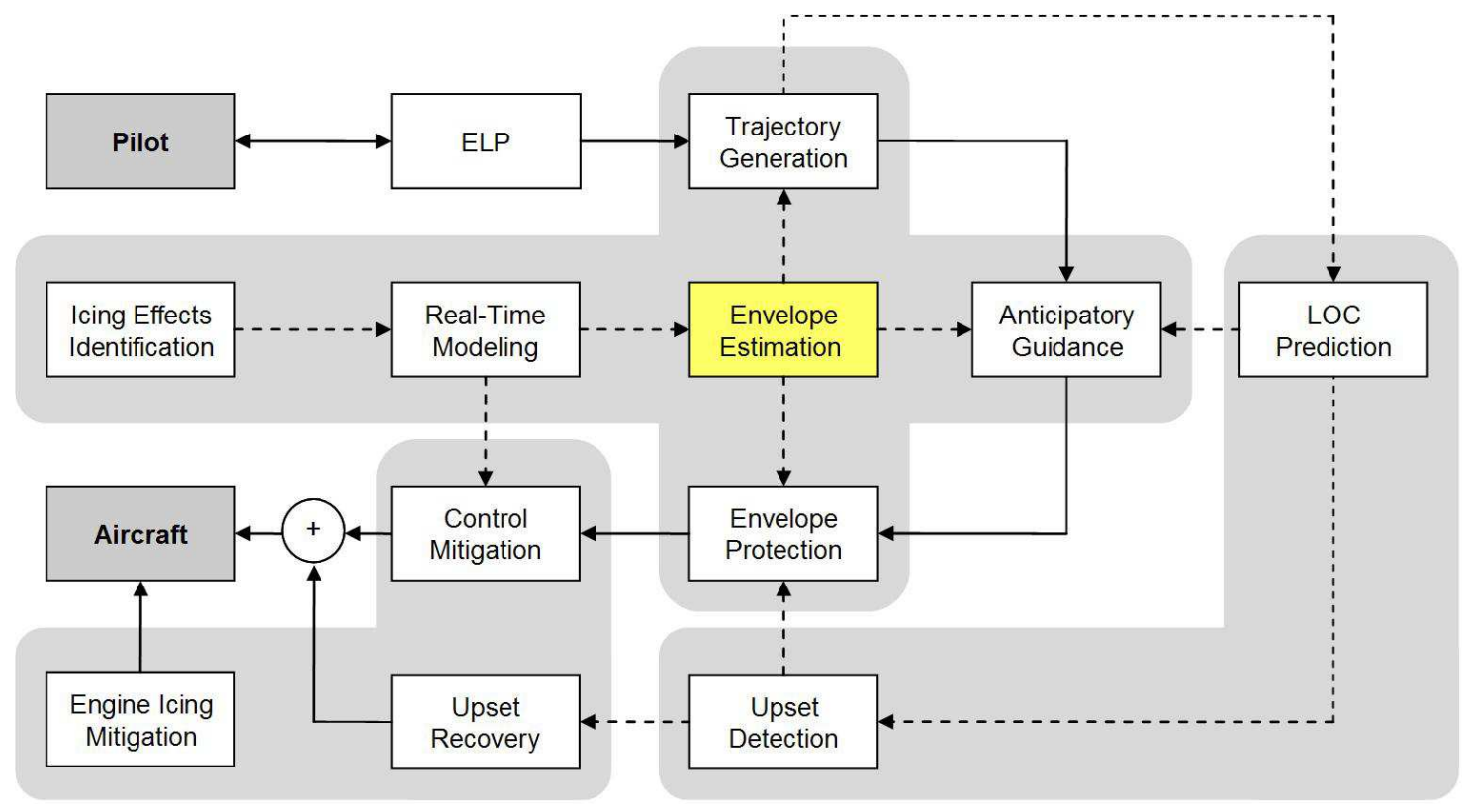

Figure 16. Overview of interconnection with other VSST components

The algorithm for estimating the maneuvering envelope relies on some information which needs to be available. First, the aerodynamic derivatives and their standard deviations need to be estimated in real time, such that up to date information is available before and after failure. With this information, a stable trim envelope needs to be determined first. The values for derivatives and standard deviations, and the boundaries of the stable trim envelope serve as a-priori information for the algorithm discussed here. The output of the algorithm is then provided to the trajectory generation and anticipatory guidance algorithms. A more detailed overview of this can be found in Fig. 17. 
Tactical Flight Management System with Maneuverability Estimation for Upset Prevention

T-FMS-ME
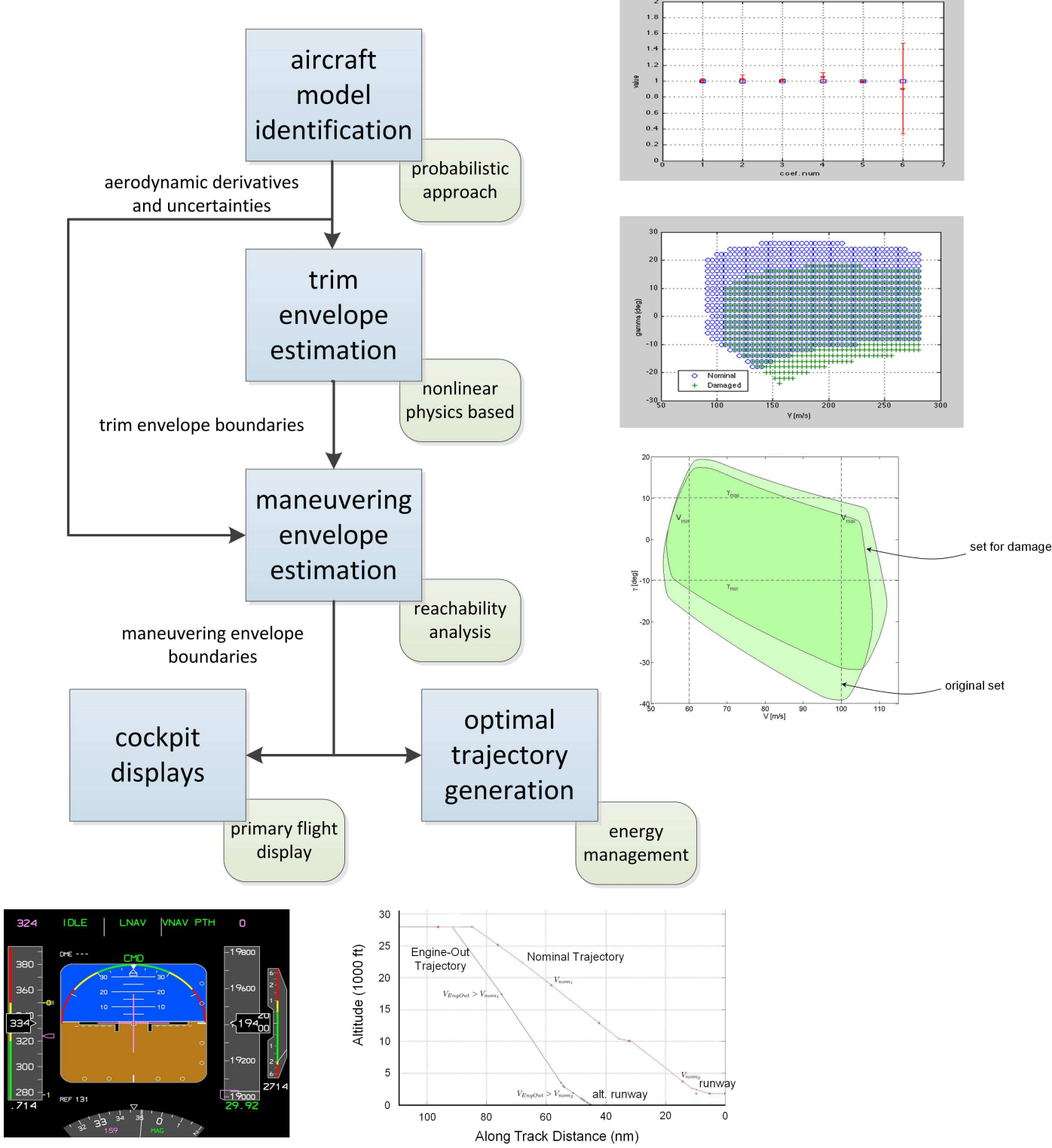

Figure 17. Detailed overview of task package maneuverability estimation and upset prevention 


\section{Conclusions and future work}

In this paper, a computationally efficient algorithm for estimating the safe maneuvering envelope of damaged aircraft has been discussed. The algorithm performs a robust reachability analysis through an optimal control formulation while making use of time scale separation and taking into account uncertainties in the aerodynamic derivatives. The safe maneuvering envelope is defined as the cross section between the forwards reachable and backwards reachable sets, which have been calculated starting from the stable trim envelope. Moreover, the backwards reachable set can be considered as the survivable maneuvering envelope, from where it is possible to bring the aircraft back to a safe trim condition after an upset due to damage, turbulence, a wake encounter etc. Damage scenario applications are included for two dimensional (in the plane of symmetry) and three dimensional examples. Results were found to be consistent with the underlying physical principles. This approach differs from others since it is physically inspired. This more transparent approach allows interpreting data in each step, and it is assumed that these physical models based upon flight dynamics theory will therefore facilitate certification for future real life applications.

At this point, only the slow dynamics as specified in Fig. 2 have been considered. Future work will extend to the faster dynamics in higher bandwidths. It should be noted that only the low bandwidth dynamics have a full nonlinear input-output description, leading to a more complex scheme to determine the optimizing control inputs. The faster dynamics for both other ranges can be represented as a linear matrix inputoutput relationship, which simplifies the optimization scheme considerably. Moreover, the middle range is aircraft independent and does not need any robustness measures for uncertainties in aerodynamic derivatives. Another topic for further research is determining the bandwidth dependent time horizon values for every sub-part of the dynamics.

\section{Acknowledgments}

The authors would like to acknowledge Kalmanje Krishnakumar, Nithin Govindarajan, Vahram Stepanyan and Jonathan Barlow for their important contributions to the research.

This research is supported by a Marie Curie International Outgoing Fellowship (IOF) within the 7th European Community Framework Program.

\section{References}

\footnotetext{
1 "Statistical Summary of Commercial Jet Airplane Accidents - Worldwide Operations 1959 - 2011," Tech. rep., Boeing Commercial Aircraft, July 2012, Available at: http://www.boeing.com/news/techissues/pdf/statsum.pdf.

${ }^{2}$ Lombaerts, T., Fault Tolerant Flight Control - A Physical Model Approach, Phd dissertation, Delft University of Technology, May 2010, Available at: http://repository.tudelft.nl/view/ir/uuid\%3A538b0174-fe84-43af-954d-02f256b2ec50/.

${ }^{3}$ Falkena, W., Borst, C., and Mulder, J., "Investigation of Practical Flight Envelope Protection Systems for Small Aircraft," Guidance, Navigation, and Control and Co-located Conferences, No. AIAA 2010-7701, American Institute of Aeronautics and Astronautics, Aug. 2010.

${ }^{4}$ Stepanyan, V., Bijl, H., Krishnakumar, K., and Barlow, J., "Adaptive Estimation Based Loss of Control Detection and Mitigation," Guidance, Navigation, and Control and Co-located Conferences, No. AIAA 2011-6609, American Institute of Aeronautics and Astronautics, Aug. 2011.

${ }^{5}$ Barlow, J., Stepanyan, V., and Krishnakumar, K., "Estimating Loss-of-Control: a Data-Based Predictive Control Approach," Guidance, Navigation, and Control and Co-located Conferences, No. AIAA 2011-6408, American Institute of Aeronautics and Astronautics, Aug. 2011.

${ }^{6}$ Govindarajan, N., An Optimal Control Approach for Estimating Aircraft Command Margin - with applications to Loss-Of-Control Prevention, Masters thesis, Delft University of Technology, October 2012, Available at: http://repository.tudelft.nl/view/ir/uuid\%3A48dbad61-728a-4c7e-ba3e-cf8382ef1cef/.

${ }^{7}$ Tang, L., Roemer, M., Bharadwaj, S., and Belcastro, C., "An Integrated Health Assessment and Fault Contingency Management System for Aircraft," AIAA Guidance, Navigation and Control Conference, No. AIAA 2008-6505, August 2008.

${ }^{8}$ Goman, M., Khramtsovsky, A., and Kolesnikov, E., "Evaluation of Aircraft Performance and Maneuverability by Computation of Attainable Equilibrium Sets," Journal of Guidance, Control and Dynamics, Vol. 31, No. 2, March - April 2008, pp. 329-339.

${ }^{9}$ Keller, J. D., McKillip, R. M., and Kim, S., "Aircraft Flight Envelope Determination using Upset Detection and Physical Modeling Methods," AIAA Guidance, Navigation and Control Conference, No. AIAA 2009-6256, August 2009.

${ }^{10}$ Boskovic, J. D., Redding, J., and Knoebel, N., "An Adaptive Fault Management (AFM) System for Resilient Flight Control," AIAA Guidance, Navigation and Control Conference, No. AIAA 2009-6263, August 2009.

${ }^{11}$ Goman, M. and Khramtsovsky, A., "Computational Framework for Investigation of Aircraft Nonlinear Dynamics," $A d-$ vances in Engineering Software, Vol. 39, 2008, pp. 367-177.
} 
${ }^{12}$ Kwatny, H. G. and Allen, R. C., "Safe Set Maneuverability of Impaired Aircraft," Guidance, Navigation, and Control and Co-located Conferences, No. AIAA 2012-4405, American Institute of Aeronautics and Astronautics, Aug. 2012.

${ }^{13}$ Menon, P., Kim, J., Sengupta, P., Vaddi, V., Yang, B.-J., and Kwan, J., "Onboard Estimation of Impaired Aircraft Performance Envelope," Guidance, Navigation, and Control and Co-located Conferences, No. AIAA 2011-6688, American Institute of Aeronautics and Astronautics, Aug. 2011.

${ }^{14}$ Lygeros, J., "On reachability and minimum cost optimal control," Automatica, Vol. 40, 2004, pp. 917-927.

${ }^{15}$ Mitchell, I. M., "The flexible, extensible and efficient toolbox of level set methods," journal of Scientific Computing, Vol. 35, 2008, pp. 300-329.

${ }^{16}$ Kwatny, H. G., Dongmo, J.-E. T., Bor-Chin Chang, G. B., Yasar, M., and Belcastro, C., "Aircraft Accident Prevention: Loss-of-Control Analysis," AIAA Guidance, Navigation and Control Conference, No. AIAA 2009-6256, August 2009.

${ }^{17}$ Tang, L., Roemer, M., Ge, J., Crassidis, A., Prasad, J., and Belcastro, C., "Methodologies for Adaptive Flight Envelope Estimation and Protection," AIAA Guidance, Navigation and Control Conference, No. AIAA 2009-6260, August 2009.

${ }^{18}$ Allen, R. C., Kwatny, H. G., and Bajpai, G., "Safe Set Protection and Restoration for Unimpaired and Impaired Aircraft," Guidance, Navigation, and Control and Co-located Conferences, No. AIAA 2012-4822, American Institute of Aeronautics and Astronautics, Aug. 2012.

${ }^{19}$ Kitsios, I. and Lygeros, J., "Launch pad Abort Flight Envelope Computation for a Personnel Launch Vehicle using Reachability," AIAA Guidance, Control and Navigation, No. AIAA 2005-6150, August 2005.

${ }^{20}$ Oort, E. V., Chu, P., and Mulder, J. A., Advances in Aerospace Guidance, Navigation and Control, chap. Maneuvering Envelope Determination through Reachability Analysis, Springer - Verlag, 2011, pp. 91-102.

${ }^{21}$ Pandita, R., Chakraborty, A., Seiler, P., and Balas, G., "Reachability and Region of Attraction Analysis applied to GTM Dynamic Flight Envelope Assessment," AIAA Guidance, Navigation and Control Conference, No. AIAA 2009-6258, August 2009.

${ }^{22}$ Bacon, B. J., "Quaternion based control architecture for determining controllcontrol/maneuverability limits," Guidance, Navigation, and Control and Co-located Conferences, No. AIAA 2012-5028, American Institute of Aeronautics and Astronautics, Aug. 2012.

${ }^{23}$ Shin, J.-Y. and Belcastro, C., "Robustness Analysis and Reliable Flight Regime Estimation of an Integrated Resilient Control System for a Transport Aircraft," AIAA Guidance, Navigation and Control Conference, No. AIAA 2008-6656, August 2008.

${ }^{24}$ Urnes, J. M., Reichenbach, E. Y., and Smith, T. A., "Dynamic Flight Envelope Assessment and Prediction," AIAA Guidance Navigaton and Control Conference, No. AIAA 2008-6983, August 2008.

${ }^{25}$ Lichter, M. D., Bateman, A. J., and Balas, G., "Flight Test Evaluation of a Rum-time Stability Margin Estimation Tool," AIAA Guidance, Navigation and Control Conference, No. AIAA 2009-6257, August 2009.

${ }^{26}$ Perhinschi, M. G., Moncayo, H., and Davis, J., "Integrated Framework for Aircraft Sub-system Failure Detection, Identification and Evaluation based on the artificial immune system paradigm," AIAA Guidance, Navigation and Control Conference, No. AIAA 2009-6261, August 2009.

${ }^{27}$ Sanchez, S. P., Perhinschi, M. G., Moncayo, H., Napolitano, M. R., Davis, J., and Fravolini, M. L., "In-Flight Actuator Failure Detection and Identification for a reduced size UAV using the artificial immune system approach," AIAA Guidance Navigation and Control Conference, No. AIAA 2009-6266, August 2009.

${ }^{28}$ Moncayo, H., Perhinschi, M., and Davis, J., "Artificial Immune System - Based Aircraft Failure Evaluation over Extended Flight Envelope," Guidance, Navigation, and Control and Co-located Conferences, No. AIAA 2010-8038, American Institute of Aeronautics and Astronautics, Aug. 2010.

${ }^{29}$ Koolstra, H. J., Damveld, H. J., and Mulder, J. A., "Envelope determination of damaged aircraft," Guidance, Navigation, and Control and Co-located Conferences, No. AIAA 2012-4699, American Institute of Aeronautics and Astronautics, Aug. 2012.

${ }^{30}$ Looye, G. and Bennani, S., "Description and Analysis of the Research Civil Aircraft Model (RCAM)," Technical report TP-088-27, Group for Aeronautical Research and Technology in Europe GARTEUR, 1997. 